\title{
Vertex-Rounding a Three-Dimensional Polyhedral Subdivision
}

\author{
S. Fortune \\ Bell Laboratories, \\ Murray Hill, NJ 07974, USA \\ sjf@ research.bell-labs.com
}

\begin{abstract}
Let $P$ be a polyhedral subdivision in $\mathbb{R}^{3}$ with a total of $n$ faces. We show that there is an embedding $\sigma$ of the vertices, edges, and facets of $P$ into a subdivision $Q$, where every vertex coordinate of $Q$ is an integral multiple of $2^{-\left\lceil\log _{2} n+2\right\rceil}$. For each face $f$ of $P$, the Hausdorff distance in the $L_{\infty}$ metric between $f$ and $\sigma(f)$ is at most $\frac{3}{2}$. The embedding $\sigma$ preserves or collapses vertical order on faces of $P$. The subdivision $Q$ has $O\left(n^{4}\right)$ vertices in the worst case, and can be computed in the same time.
\end{abstract}

\section{Introduction}

Geometric algorithms are usually described in the "real-number RAM" model of computation, where arithmetic operations on real numbers have unit cost. A programmer implementing a geometric algorithm must find some substitution for real arithmetic. The substitution of exact arithmetic on a subset of the reals, say the integers or the rationals, avoids the difficulties that can arise from naive substitution of floating-point arithmetic [4], [12], [14], [15]. The substitution is not trivial, since the required arithmetic bit-length usually exceeds the native arithmetic bit-length of most computer hardware, and some form of software arithmetic is required.

Recent research has made the use of software exact arithmetic for geometric algorithms much more attractive. A predicate on geometric data is determined by the sign of an arithmetic expression in the coordinates of the data. A promising strategy for sign-evaluation is adaptive-precision arithmetic [6], [13], [20], where the expression is evaluated to higher and higher precision until its sign is known, i.e., until the magnitude of the expression exceeds an error bound. Low precision, even floating-point, suffices most of the time, since most instances of geometric predicates are easy. In addition, for some basic predicates like the sign of a determinant, there are alternative evaluation strategies that require arithmetic with relatively low precision [1]-[3]. 
Exact arithmetic would be more useful if high-level geometric rounding algorithms were available. Virtually any geometric construction that produces new geometric data increases the bit-length of geometric coordinates. For example, suppose points are represented with homogeneous integer coordinates. The plane through three such points has coefficients whose bit-lengths are about three times the point coordinate bit-lengths; the point of intersection of three such planes has coordinate bit-length about nine times that of the original points. Thus a solid modeler, which implements boolean operations and rigid motions on polyhedra, might produce a polyhedron with high coordinate bit-length even if the original polyhedra had short coordinate bit-length. Typically an application requires only a low-precision approximation, not the exact answer. Hence there is a need for high-level rounding, which replaces a geometric structure with high bit-length coordinates with an approximating structure with short bit-length coordinates. It does not suffice to round each coordinate independently, since such rounding is a geometric perturbation, and may introduce inconsistencies between geometric and combinatorial information. Furthermore, some change in combinatorial structure is inevitable; indeed, in certain cases it is NP-hard to determine if it is possible to round to low-precision without changing combinatorial structure [19].

Satisfactory high-level rounding algorithms are known for polygonal subdivisions in two dimensions. One such algorithm is snap-rounding [10]. Fix a polygonal subdivision, with arbitrary-precision coordinates. A pixel is a unit square in the plane centered at a point with integer coordinates; a pixel is hot if it contains a vertex of the subdivision. Snap-rounding replaces each vertex by the center of the pixel containing the vertex, and each edge by the polygonal chain through the centers of the hot pixels met by the edge, in the same order as met by the edge. The snap-rounded subdivision approximates the original subdivision in the sense that each vertex and edge of the original subdivision has an image in the snap-rounded arrangement whose Hausdorff distance is at most $\frac{1}{2}$ in the $L_{\infty}$ metric. Snap-rounding may change the combinatorial structure of the subdivision, for example, vertices and edges may collapse together, but some combinatorial ordering information is preserved [10].

This paper presents a generalization of snap-rounding to polyhedral subdivisions in three dimensions. Fix a polyhedral subdivision $P$ with a total of $n$ vertices, edges, and facets. We show that there is a polyhedral subdivision $Q$ so that each vertex coordinate is an integer multiple of $1 / 2^{\left\lceil\log _{2} n\right\rceil+2}$. Each face $f$ of $P$ has an image $\sigma(f)$ in $Q$ so that the Hausdorff distance between $f$ and $\sigma(f)$ is at most $\frac{3}{2}$. As with snap-rounding in two dimensions, $f$ and $\sigma(f)$ may have different combinatorial structures: an edge may be replaced with a polygonal chain, and a facet with a triangulation. Two vertices may collapse together; the polygonal chains for two edges or the triangulations for two facets may collapse together or overlap partially, perhaps in several places. However, vertical order is preserved (or collapsed): if face $f$ is vertically above face $f^{\prime}$ (i.e., there is a line parallel to the $z$-axis meeting both faces, and the intersection with $f$ has higher $z$-coordinate), then $\sigma(f)$ is above (or overlaps) $\sigma\left(f^{\prime}\right)$. In the worst case $Q$ has $O\left(n^{4}\right)$ vertices and can be computed in time $O\left(n^{4}\right)$.

As is the case with snap-rounding in two dimensions, the Hausdorff distance between a facet $f$ and its image $\sigma(f)$ can be reduced by scaling coordinates. For example, for $k>0$, the Hausdorff distance can be reduced to at most $\frac{3}{2} \cdot 2^{-k}$, by multiplying every coordinate of $P$ by $2^{k}$, rounding as above, and then dividing every coordinate of the result 
by $2^{k}$. Of course, coordinates are now integral multiples of $1 / 2^{k+\lceil\log n\rceil+2}$. Alternatively, the same procedure with $k=-(\lceil\log n\rceil+2)$ guarantees an approximating subdivision with integer coordinates, although with Hausdorff distance bounded by $6 n$.

Though the algorithm in this paper demonstrates the theoretical possibility of threedimensional snap-rounding, it is not immediately practical. One concern is the discouragingly large bound on the number of new vertices. The algorithm as described always adds all vertices that might potentially be needed; a variant algorithm might add vertices only as necessary. It is plausible that for typical subdivision instances-not chosen by an adversary - the number of new vertices will be acceptable. Another concern is the complexity of the algorithm. Snap-rounding in two dimensions is essentially a local algorithm, with the rounding of each vertex and edge determined simply from the set of hot pixels. Unfortunately, as is seen below, the three-dimensional algorithm requires more complicated global information. Devising a simple, practical, and efficient three-dimensional rounding algorithm is a significant open problem.

Other Work. Greene and Yao were the first to suggest a rounding scheme for polygonal subdivisions in two dimensions [8]. Hobby [11] and Greene [9] give algorithms to compute the snap-rounding of the arrangement formed by a set of intersecting edges. Guibas and Marimount [10] show how to maintain the snap-rounded arrangement of edges under insertion and deletion of edges; they also give elementary proofs of basic topological properties of snap-rounding. Goodrich et al. [7] give improved algorithms to snap-round a set of intersecting edges, in the case when there are many intersections within a pixel. Milenkovic [18] suggests a "shortest-path" geometric rounding scheme that sometimes introduces fewer bends than snap-rounding.

Goodrich et al. [7] propose a scheme for snap-rounding a set of edges in three dimensions after first adding as vertices the points of "closest encounter" between nearby edges. Milenkovic [16] sketches a scheme for rounding a polyhedral subdivision in three dimensions (in fact, any dimension). Unfortunately, both schemes have the property that rounded edges can cross (see below), which violates any notion of topological consistency.

Fortune [5] suggests a high-level rounding algorithm for polyhedra in three dimensions. His algorithm assumes that a polyhedron is presented by the equations of its face planes (and the combinatorial incidence structure of faces), not the coordinates of vertices as assumed by snap-rounding. His algorithm does not appear to extend from polyhedra to polyhedral subdivisions.

The Challenges of Three-Dimensional Snap-Rounding. The obvious way to snap-round a vertex in three dimensions is to replace it with the center of the voxel containing it. (A voxel is a unit cube centered at an integer point.) It is less clear how to snap-round edges and facets.

Snap-rounding a set of edges in three dimensions requires the addition of new vertices, unlike the situation in two dimensions. Consider two transverse nearby edges. Rounding the endpoints to voxel centers perturbs the edges, and hence the edges may change orientation or cross. We can attempt to prevent this by adding a vertex in the interior of each edge near the other edge; then either the two new vertices are in the same voxel and snap-round together, or they are in different voxels and the snap-rounded edges will 


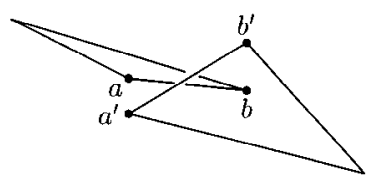

Fig. 1. Vertices $a$ and $a^{\prime}$ project to the same pixel in the $x y$-plane, as do $b$ and $b^{\prime}$. Hence in three dimensions, the snap-rounding of $a b$ crosses the snap-rounding of $a^{\prime} b^{\prime}$.

not cross. Clearly, it might be necessary to add quadratically many vertices, if the edges form a "cross-hatch" pattern.

Snap-rounding with facets as well is more problematic. If a vertex $v$ and a facet $f$ are nearby, we can add a new vertex $v^{\prime}$ to $f$ to ensure that $v$ and $f$ are properly separated or collapsed. However, this requires that $f$ be triangulated, which introduces new edges. Potentially these edges are close to old edges, which could require new vertices, and it is not immediate that the process is finite. We can attempt to ensure termination by projecting nearby edges onto a facet, and then triangulating the facet compatibly with the projection. The actual rounding algorithm is a formalization of this idea.

Overview of the Rounding Algorithm. The rounding algorithm is based on the following general outline. Orthogonally project all edges of the subdivision $P$ onto the $x y$-plane, form the arrangement, snap-round, and compute a triangulation $T$. Each facet $f$ of $P$ has an image $T_{f}$ that forms a subtriangulation $T_{f}$ of $T$. The rounding of facet $f$ is obtained by lifting $T_{f}$ to a polygonal surface $\sigma(f)$ that approximates $f$. By considering each cylinder over a vertex, edge, or triangle of $T$ separately, we can ensure that the lifting preserves (or collapses) the vertical order on faces of $P$.

The first step of the actual algorithm is to determine the rounding $\sigma(e)$ of each edge $e$ of $P$; recall that $\sigma(e)$ in general can be a polygonal chain. This step is nontrivial, since we must prevent crossings among the resulting edges (see Fig. 1). To prevent crossings, we subdivide the edges of $P$ by all $x y-, x z^{-}$, and $y z$-intersection points. (If the orthogonal projections of $e$ and $e^{\prime}$ into the $x z$-plane cross at a point $p$, and $l$ is the line parallel to the $y$-axis through $p$, then $e \cap l$ and $e^{\prime} \cap l$ are $x z$-intersection points.) While this subdivision prevents most crossings, it is not quite sufficient to prevent all crossings. In Fig. 2 the

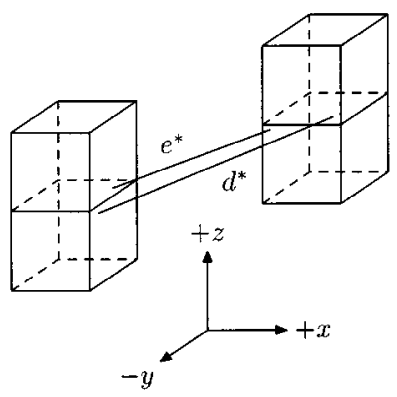

Fig. 2. The endpoints of $e^{*}$ and $d^{*}$ lie on column boundaries (or extend slightly inside). The roundings $\rho\left(d^{*}\right)$ and $\rho\left(e^{*}\right)$ cross, although the $x y-, x z-$, and $y z$-projections of $d^{*}$ and $e^{*}$ do not. 


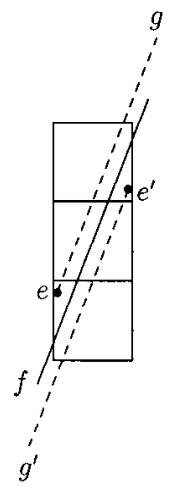

Fig. 3. Side view. Edge $e$ is above facet $f$ and $e^{\prime}$ is below. Hence the rounding of facet $f$ must contain the vertical interval from the rounding of $e$ to the rounding of $e^{\prime}$.

$x y$-, $x z-$, and $y z$-projections of $d^{*}$ and $e^{*}$ are all disjoint, but their snap-roundings cross. Fortunately, the configuration of Fig. 2 is almost the only way this can happen, and we can show that there is a slight modification of snap-rounding that does avoid crossings. For example, in Fig. 2 the modified snap-rounding of $d^{*}$ is a two-edge polygonal chain, connecting a snap-rounded endpoint of $d^{*}$ to the snap-rounded endpoint of $e^{*}$ on the same vertical line, and then to the other snap-rounded endpoint of $d^{*}$. We show that the distance between an edge and its modified snap-rounding increases slightly, to at most $\frac{3}{2}$. (The configuration in Fig. 2 can be modified to show that the "close encounter" subdivision of Goodrich et al. [7] does not prevent edge crossings.) Section 4 below describes the subdivision and modification of snap-rounding.

The second step of the actual algorithm is to lift $T_{f}$ to its image $\sigma(f)$. The lifting must respect constraints on vertical order among facets and edges; for example, if facet $f$ is vertically above edge $e$, then $\sigma(f)$ must be above or contain $\sigma(e)$. These constraints can be somewhat complex. In the schematic view in Fig. 3, facet $f$ is below edge $e$ of facet $g$ and above edge $e^{\prime}$ of facet $g^{\prime}$. If $\sigma(e)$ and $\sigma\left(e^{\prime}\right)$ have the same $x y$-projection with $\sigma\left(e^{\prime}\right)$ above $\sigma(e)$, then $\sigma(f)$ must contain the entire vertical interval between $\sigma(e)$ and $\sigma\left(e^{\prime}\right)$ (as do $\sigma(g)$ and $\sigma\left(g^{\prime}\right)$, in this case).

The lifting $\sigma(f)$ is determined by merging the lifted images of each vertex, edge, and triangle of $T_{f}$. For a vertex $v$ of $T_{f}$, its lifting $l_{f}(v)$ may just be a vertex; however, it could be a vertical chain of edges if there are vertices $v^{\prime}$ and $v^{\prime \prime}$ of $P$ so that $v^{\prime}$ is above $f, v^{\prime \prime}$ is below $f, \sigma\left(v^{\prime}\right)$ is below $\sigma\left(v^{\prime \prime}\right)$, and both $\sigma\left(v^{\prime}\right)$ and $\sigma\left(v^{\prime \prime}\right)$ project and snap-round to $v$. Similarly, the lifting $l_{f}(e)$ of an edge $e$ of $T_{f}$ may just be an edge or it may contain the vertical interval between two edges whose $x y$-projection snap-rounds to $e$.

The lifting $l_{f}(\Delta)$ of a triangle $\Delta$ of $T_{f}$ is more complicated. It is defined in terms of the lifting $l_{f \Delta}(e)$ for each edge $e$ of $\Delta$. The lifting $l_{f \Delta}(e)$ is just an edge with $x y$-projection $e$; it will form part of the boundary of $l_{f \Delta}(e)$. It must satisfy three properties: it must be close to $f$, it must not cross any other lifted edge, and it must respect vertical order with other lifted edges (i.e., if $f$ is vertically below $f^{\prime}$, then $l_{f \Delta}(e)$ must be below $l_{f^{\prime} \Delta}(e)$ ). The last property is crucial to establishing that the lifted triangles $l_{f}(\Delta)$ respect vertical order. Satisfying all three properties requires some care (see Section 5). 


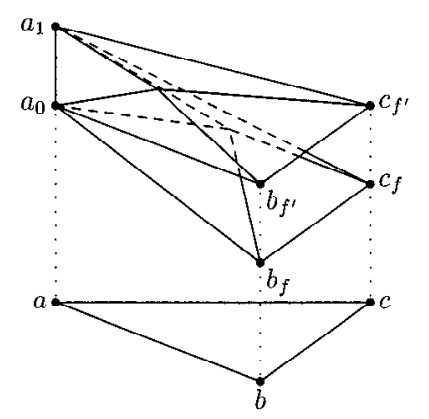

Fig. 4. The liftings of triangle $a b c$ for facets $f$ and $f^{\prime}$ have boundary $a_{1} a_{0} b_{f} c_{f}$ and $a_{1} a_{0} b_{f^{\prime}} c_{f^{\prime}}$, respectively.

There are two naively plausible properties of the definition of $l_{f \Delta}$ that do not hold. First, if $\Delta$ and $\Delta^{\prime}$ are both incident to $e$ and in $T_{f}$, then there is no guarantee that $l_{f \Delta}(e)=l_{f \Delta^{\prime}}(e)$ (though they do not cross). However, this causes no difficulty (since $l_{f}(e)$ contains both $l_{f \Delta}(e)$ and $\left.l_{f \Delta^{\prime}}(e)\right)$.

The second untrue property causes more difficulty. Suppose $\Delta$ has vertices $a, b, c$. There is no guarantee that, say, $l_{f \Delta}(a b)$ is incident to $l_{f \Delta}(a c)$ (though both meet the vertical line through $a$ ). Hence $l_{f}(\Delta)$ must be a triangulation of the polygon formed by $l_{f \Delta}(a b), l_{f \Delta}(b c)$, and $l_{f \Delta}(a c)$, and perhaps edges along the vertical lines through $a$, $b$, and $c$. See Fig. 4 . It is easy to triangulate the polygon using a central vertex whose $x y$-projection is within triangle $\Delta$. However, a vertical boundary edge may be shared among several different liftings. To ensure that there are no crossings among edges, each central vertex must have distinct coordinates. Since there may be $\Omega(n)$ central vertices, coordinates that are integer multiples of roughly $1 / n$ are necessary. This leads to the additional $\lceil\log n\rceil+2$ bits needed for vertex coordinates. More details of the lifting appear in Section 6.

Naively the rounded subdivision $Q$ has at most $O\left(n^{3}\right)$ faces: the triangulation $T$ has $O\left(n^{2}\right)$ triangles, so for each facet $f$ the rounding $\sigma(f)$ consists of $O\left(n^{2}\right)$ lifted triangles $\left\{l_{f}(\Delta)\right\}$. However, in the worst case each lifted triangle $l_{f}(\Delta)$ may consist of $O(n)$ faces, since there could be linearly many vertices on the vertical edges of its boundary. Hence $Q$ has $O\left(n^{4}\right)$ faces.

\section{The Main Theorem}

For points $a, b \in \mathbb{R}^{3}$ and sets $A, B \subset \mathbb{R}^{3}, d(a, b)$ is the $L_{\infty}$ distance between $a$ and $b$ (the $L_{\infty}$ distance is used exclusively in this paper); $d(a, B)$ is $\inf _{b \in B} d(a, b)$; and $d(A, B)$ is $\sup _{a \in A} d(a, B)$. Note that $d$ is symmetric for points, but not in general for sets. The Hausdorff distance $d_{\mathrm{H}}(A, B)$ is $\max (d(A, B), d(B, A))$.

The direction parallel to the $z$-axis is the vertical direction. Two sets $A, B \subset \mathbb{R}^{3}$ are vertically ordered $A \prec B$ (read " $A$ is below $B$ ") if there is a vertical line meeting both $A$ and $B$, and, for every vertical line $l$ meeting $A$ and $B, A \cap l$ is below $B \cap l$, i.e., the $z$-coordinate of every point of $A \cap l$ is less than the $z$-coordinate of every point in $B \cap l$. 
Sets $A$ and $B$ satisfy $A \preceq B$ if there is a vertical line meeting both, and, for every vertical line meeting both, $A \cap l$ is below or intersects $B \cap l$. As is well known, $\prec$ is not transitive in general; it is transitive among a family of sets that have the same $x y$-projection. If furthermore every family in the set is a surface, i.e., every vertical line misses the set or meets it at one point, then $\preceq$ is transitive.

A subdivision $P$ in $\mathbb{R}^{3}$ is a set of compact convex polyhedral cells so that every face of every cell is in the subdivision and so that the intersection of two cells is a face of both. Cells of dimension 0,1 , and 2 are vertices, edges, and facets, respectively. $|P|$ is the union of the cells of $P$. An embedding of a subdivision $P$ into a subdivision $Q$ is a mapping $\sigma$ that maps each cell of $P$ into a subdivision contained in $Q$ so that if $f$ is a face of $f^{\prime}$, then $\sigma(f) \subseteq \sigma\left(f^{\prime}\right)$.

To simplify notation somewhat, we extend $d$ and $\prec$ to subdivisions. Thus for subdivisions $P$ and $Q, P \prec Q$ means $|P| \prec|Q|$ and $d(P, Q)$ means $d(|P|,|Q|)$.

Throughout this paper we assume that subdivisions in $\mathbb{R}^{3}$ do not include cells of dimension 3. Furthermore, we assume that every subdivision is in general position, specifically, that no edge or facet is parallel to a coordinate axis and that no vertex has a coordinate that is an integer multiple of $\frac{1}{2}$. The general position assumption simplifies presentation; it is not hard to remove (either explicitly or for example by an infinitesimal symbolic rigid motion).

Theorem 2.1. Let $P$ be a subdivision in $\mathbb{R}^{3}$ with a total of $n$ cells; set $\kappa=\frac{3}{2}$. There is a subdivision $Q$ and an embedding $\sigma$ of $P$ into $Q$ so that:

(1) For each cell $f$ of $P, d_{\mathrm{H}}(f, \sigma(f))<\kappa$.

(2) Each vertex coordinate of $Q$ is an integral multiple of $1 / 2^{\left\lceil 2+\log _{2} n\right\rceil}$.

(3) If cells $f, f^{\prime}$ of $P$ satisfy $f \preceq f^{\prime}$, then $\sigma(f) \preceq \sigma\left(f^{\prime}\right)$.

(4) $Q$ can be computed in time $O\left(n^{4}\right)$ and has $O\left(n^{4}\right)$ cells.

This theorem follows from the discussion below, in particular Lemmas 6.2, 6.5, 6.6, and Corollary 7.4 below. At a high level, the algorithm required for step (4) has three steps.

1. Subdivide the vertices and edges of $P$, forming a set of vertices and edges $P^{*}$ (Section 4).

2. Orthogonally project $P^{*}$ onto the $x y$-plane, snap-round, and triangulate the convex hull of the resulting subdivision. Let $T$ be the resulting triangulation.

3. For each cell $f$ in $P$, lift $T_{f}$ (the image of $f$ in $T$ ) to a subdivision $Q_{f} \subset \mathbb{R}^{3}$ (Section 6).

\section{Definitions}

There are many symbols defined in this paper. For reference, most are summarized in Appendix 8.

A pixel is an open unit square in the $x y$-plane centered at an integer point; $\operatorname{pixel}(q)$ is the pixel containing point $q$. A voxel is an open unit cube in $\mathbb{R}^{3}$ centered at an integer point; $\operatorname{voxel}(q)$ is the voxel containing point $q$. A column (of voxels) is all voxels whose centers have the same $x$ - and $y$-coordinates; $\operatorname{column}(q)$ is the column containing $q$. 
Let $A$ be a subdivision in the $x y$-plane. A pixel is hot (with respect to $A$ ) if it contains a vertex. The snap-rounding (with respect to $A$ ) of an edge $e$ of $A$ is the polygonal chain connecting the centers of the hot pixels met by $e$ in the same order as met by $e$; similarly, the snap-rounding of a vertex of $A$ is the center of the hot pixel containing it. A basic fact $[10]$ is that two polygonal chains that result from snap-rounding intersect only at vertices and edges of both chains. The snap-rounding of $A$ is obtained by replacing each edge and vertex of $A$ with its snap-rounding with respect to $A$; it is a polygonal subdivision whose vertices are hot pixel centers, i.e., integer points, and whose edges connect integer points.

Let $\pi_{x y}$ be orthogonal projection onto the $x y$-plane, and similarly for $\pi_{x z}$ and $\pi_{y z}$. A set $A \subset \mathbb{R}^{3}$ is over a set $P$ in the $x y$-plane if $\pi_{x y}(A)=P$. If $A$ is a surface with $p \in \pi_{x y}(A)$, then $A_{p}$ is the point of $A$ over $p$ (i.e., $\pi_{x y}\left(A_{p}\right)=p$ ). If $A$ and $B$ are surfaces over the same set, then $\max (A, B)$ is the pointwise maximum (viewed as functions of the $x y$-plane), and $\min (A, B)$ is the pointwise minimum. If $A, B, C$ are surfaces over the same set with $A \succeq B$, then $\operatorname{snap}(C,[A, B])$ is $\min (A, \max (B, C))$. Clearly, $A \succeq \operatorname{snap}(C,[A, B]) \succeq B$.

Suppose a set $P$ in the $x y$-plane is fixed. We define symbolic sets $\top$ (top) and $\perp$ (bottom) satisfying $\perp \prec A \prec \top$ for any other set $A$ over $P$. We have for example $\min (A, \top)=A=\max (A, \perp)$; we define min and max of an empty collection to be $\top$ and $\perp$, respectively.

Two edges cross if they intersect at a point interior to at least one of the edges.

Proposition 3.1. Suppose $T \subset \mathbb{R}^{3}$ is convex, $\left\{s_{1}, \ldots, s_{k}\right\} \subset \mathbb{R}^{3}$ is a finite set of points with convex hull $S$, and $\kappa \geq 0$. If $d\left(s_{i}, T\right) \leq \kappa$ for $i=1, \ldots, k$, then $d(S, T) \leq \kappa$.

Proof. Any point in $S$ can be expressed as $\sum \alpha_{i} s_{i}$ with $0 \leq \alpha_{i} \leq 1$ and $\sum \alpha_{i}=1$. For each $s_{i}$, there is a point $t_{i} \in T$ so that $d\left(s_{i}, t_{i}\right) \leq \kappa$. Clearly, $\sum \alpha_{i} t_{i} \in T$ and $d\left(\sum \alpha_{i} s_{i}, \sum \alpha_{i} t_{i}\right)$ is the maximum absolute value of any coordinate of $\sum \alpha_{i}\left(s_{i}-t_{i}\right)$, which is bounded by $\kappa$ since $\sum \alpha_{i}=1, \alpha_{i} \geq 0$, and the absolute value of each coordinate of $s_{i}-t_{i}$ is bounded by $\kappa$.

\section{Snap-Rounding Edges}

Define $\rho(q)$ to be the center of the voxel containing $q$, and extend $\rho$ to edges: $\rho\left(q q^{\prime}\right)$ is the edge $\rho(q) \rho\left(q^{\prime}\right)$. The mapping $\rho$ is the obvious extension of snap-rounding to three dimensions (ignoring snapping to hot voxels, which is unimportant here). Unfortunately, $\rho$ may cause two edges to cross. We now define a refinement $P^{*}$ of the vertices and edges of $P$ and a modification $\tau$ of $\rho$ so that no two edges in $\tau\left(P^{*}\right)$ cross.

\subsection{The Subdivision $P^{*}$}

Let $e$ and $e^{\prime}$ be two edges of $P$ whose $x y$-projections cross at a point $p$. An $x y$-intersection point (of $P$ ) is either point on $e$ or $e^{\prime}$ that meets the line through $p$ parallel to the $z$-axis. The definition of an $x z$ - or $y z$-intersection point is similar. 
The subdivision $P^{*}$ results from subdividing the edges of $P$. At any point in the process, $\hat{e}$ denotes the subdivision of edge $e$ of $P$; any voxel containing a vertex is a hot voxel; and any column of voxels containing a hot voxel is a hot column. There are two steps in the subdivision:

1. Subdivide the edges of $P$ at all $x y$-, $x z-$, and $y z$-intersection points of $P$.

2. For each edge $e$ of $P$, split $\hat{e}$ by each hot column $C$ it meets: $\hat{e}$ must meet $C$ in a consecutive set of voxels; $\hat{e}$ is split by $C$ by further subdividing $\hat{e}$ at any point in the first voxel (if $\hat{e}$ does not yet have a vertex in the first voxel) and similarly by subdividing $\hat{e}$ in the last voxel.

Splitting by hot columns has an easy consequence: for any edge $e$ of $P$, the snaprounding of $\pi_{x y}(\hat{e})$ with respect to $\pi_{x y}\left(P^{*}\right)$ is identical to the snap rounding of $\pi_{x y}(\hat{e})$ with respect to $\hat{e}$. Henceforth we use a superscript “*” for edges and vertices of $P^{*}$. For $e^{*}$ an edge of $P^{*}$, we write $s\left(e^{*}\right)$ for the snap-rounding of $\pi_{x y}\left(e^{*}\right)$. It is immediate that if $d^{*}, e^{*}$ are edges of $P^{*}$, then $\rho\left(d^{*}\right)$ crosses $\rho\left(e^{*}\right)$ only if $s\left(d^{*}\right)=s\left(e^{*}\right)$.

Lemma 4.1. $P^{*}$ has $O\left(n^{3}\right)$ vertices; there are $O\left(n^{2}\right)$ hot columns and $O\left(n^{3}\right)$ hot voxels.

Proof. Clearly, there are at most $O\left(n^{2}\right) x y-, x z^{-}$, and $y z$-intersection points, and only $O(n)$ vertices of $P$. Splitting edges by hot columns adds no new hot columns, hence there are $O\left(n^{2}\right)$ hot columns. For each edge $e$ of $P$ and for each hot column, there are at most two vertices added when $\hat{e}$ is split by the column. Hence there are $O\left(n^{3}\right)$ vertices altogether.

As mentioned earlier, $T$ is a triangulation of the convex hull of $s\left(P^{*}\right)$. Consider the edges $E^{*}$ in $P^{*}$ bounding a facet $f$ of $P$. The projection $\pi_{x y}\left(E^{*}\right)$ forms a simple cycle, but the snap-rounding $s\left(E^{*}\right)$ need not. However, it is not hard to see that $s\left(E^{*}\right)$ consists of some number of simple cycles connected by polygonal chains. Let $T_{f}$ be the subtriangulation of $T$ consisting of the vertices and edges of $s\left(E^{*}\right)$ plus any vertices, edges, and triangles of $T$ interior to the simple cycles in $s\left(E^{*}\right)$.

For $v$ a vertex of $T, e$ an edge of $T$, and $\Delta$ a triangle of $T$, define

$$
\begin{aligned}
P_{e}^{*} & =\left\{e^{*} \in P^{*}: s\left(e^{*}\right)=e\right\}, \\
P_{v}^{*} & =\left\{v^{*} \in P^{*}: s\left(v^{*}\right)=v\right\}, \\
F_{e} & =\left\{f \in P^{*}: e \in T_{f}\right\}, \\
F_{\Delta} & =\left\{f \in P^{*}: \Delta \in T_{f}\right\},
\end{aligned}
$$

where $v^{*}$ and $e^{*}$ are vertices and edges of $P^{*}$, respectively, and $f$ is a facet of $P$.

\subsection{The Mapping $\tau$}

Lemma 4.2. Let $e$ be an edge of $T$. If $d^{*}, e^{*} \in P_{e}^{*}$ and $\rho\left(d^{*}\right), \rho\left(e^{*}\right)$ cross, then either there is an endpoint $w$ of $\rho\left(d^{*}\right)$ with $d\left(w, e^{*}\right)<\kappa$ or an endpoint $w^{\prime}$ of $\rho\left(e^{*}\right)$ with $d\left(w^{\prime}, d^{*}\right)<\kappa$. 
The proof of this lemma is rather intricate, so it is deferred to Appendix A.

Lemma 4.3. Let e be an edge of $T$. There is a mapping $\tau$ defined on $P_{e}^{*}$ so that:

(1) For all edges $e^{*} \in P_{e}^{*}, \tau\left(e^{*}\right)$ is an edge over e with endpoints among the endpoints of $\rho\left(P_{e}^{*}\right)$.

(2) For all edges $e^{*}, d\left(\tau\left(e^{*}\right), e^{*}\right)<\kappa$.

(3) $\tau\left(P_{e}^{*}\right)$ is noncrossing.

(4) $\tau$ can be computed in time quadratic in the size of $P_{e}^{*}$.

Proof. We define $\tau$ inductively, adding edges of $P_{e}^{*}$ one by one in arbitrary order. The addition of an edge may change the definition of $\tau$ on other edges as well; however, properties (1)-(3) of the lemma statement are maintained. For the following, recall that $A_{p}$ is the point of $A$ that has $x y$-projection $p$.

So suppose $\tau$ has been defined on a subset $S$ of $P_{e}^{*}$ and $e^{*}$ is the next edge. If no edge of $\tau(S)$ crosses $\rho\left(e^{*}\right)$, then simply define $\tau\left(e^{*}\right)=\rho\left(e^{*}\right)$. Otherwise, since $\tau(S)$ is noncrossing, we can assume up to a symmetric argument that every edge $\tau\left(d^{*}\right)$ crossing $\rho\left(e^{*}\right)$ has $\tau\left(d^{*}\right)_{u} \succ \rho\left(e^{*}\right)_{u}$ and $\tau\left(d^{*}\right)_{v} \prec \rho\left(e^{*}\right)_{v}$.

Let $q$ be the highest (in $\prec$ ) endpoint over $u$ of an edge in $\tau(S) \cup\left\{\rho\left(e^{*}\right)\right\}$ so that $d\left(q, e^{*}\right)<\kappa$; similarly let $r$ be the lowest endpoint over $v$ of an edge in $\tau(S) \cup\left\{\rho\left(e^{*}\right)\right\}$ so that $d\left(q, e^{*}\right)<\kappa$. Clearly, $\rho\left(e^{*}\right)_{u} \preceq q$ and $r \preceq \rho\left(e^{*}\right)_{v}$. If it is is possible to choose $q^{\prime}$ over $u$ in the interval $\rho\left(e^{*}\right)_{u} \cdots q$ and $r^{\prime}$ over $v$ in the interval $r \cdots \rho\left(e^{*}\right)_{v}$ so that $q^{\prime} r^{\prime}$ does not cross an edge of $\tau(S)$, define $\tau\left(e^{*}\right)$ to $q^{\prime} r^{\prime}$. Note that it is always possible to choose $q^{\prime}$ and $r^{\prime}$ among the endpoints of $\tau(S) \cup\left\{\rho\left(e^{*}\right)\right\}$ and that the distance from $q^{\prime}$ and $r^{\prime}$ to $e^{*}$ is less than $\kappa$.

Otherwise some subset $S^{\prime}$ of the edges in $S$ crosses $q r$. Clearly, for any $d^{*} \in S^{\prime}$, $\tau\left(d^{*}\right)_{u} \succ q$ and $\tau\left(d^{*}\right)_{v} \prec r$. See Fig. 5.

We claim that, for any edge $\tau\left(d^{*}\right) \in S^{\prime}$, either $d\left(q, d^{*}\right)<\kappa$ or $d\left(r, d^{*}\right)<\kappa$. If $\rho\left(d^{*}\right)_{u} \preceq q$, then since $q \preceq \tau\left(d^{*}\right)_{u}$, certainly $d\left(q, d^{*}\right)<d\left(\tau\left(d^{*}\right)_{u}, d^{*}\right)<\kappa$. Similarly if $\rho\left(d^{*}\right)_{v} \succeq r$, then $d\left(r, d^{*}\right)<\kappa$. Otherwise $\rho\left(d^{*}\right)_{u} \succ q \succeq \rho\left(e^{*}\right)_{u}$ and $\rho\left(d^{*}\right)_{v} \prec r \preceq$ $\rho\left(e^{*}\right)_{v}$, so $\rho\left(d^{*}\right)$ crosses $\rho\left(e^{*}\right)$. See Fig. 5. The hypothesis of Lemma 4.2 holds with $d^{*}$ and $e^{*}$. By the definitions of $q$ and $r, d\left(\rho\left(d^{*}\right)_{u}, e^{*}\right) \geq \kappa$ and $d\left(\rho\left(d^{*}\right)_{v}, e^{*}\right) \geq \kappa$. Hence Lemma 4.2 implies either $d\left(\rho\left(e^{*}\right)_{v}, d^{*}\right)<\kappa$ or $d\left(\rho\left(e^{*}\right)_{u}, d^{*}\right)<\kappa$, so either $d\left(q, d^{*}\right)<\kappa$ or $d\left(r, d^{*}\right)<\kappa$.

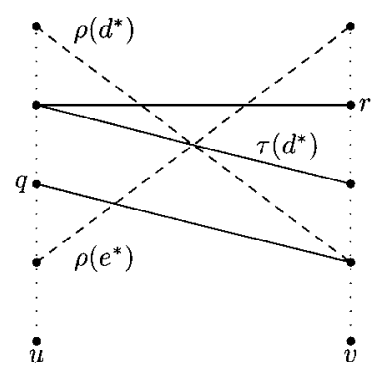

Fig. 5. Definition of $\tau$ on new edge $e^{*}$. 
Let $Q$ be the set of edges $d^{*} \in S^{\prime}$ so that $d\left(q, d^{*}\right)<\kappa$, and $R=S^{\prime} \backslash Q$. Define $\tau\left(e^{*}\right)=q r$; for $d^{*} \in Q$, redefine $\tau\left(d^{*}\right)_{u}=q$; and, for $d^{*} \in R$, redefine $\tau\left(d^{*}\right)_{v}=r$. It is easy to check that $\tau$ satisfies conditions (1)-(3). The running time is immediate.

Henceforth we let $\tau$ be defined on all edges of $P^{*}$, by choosing a definition on $P_{e}^{*}$ separately for each edge $e$ of $T$, using Lemma 4.3. Since there can be $O\left(n^{2}\right)$ edges $e$ in $T$, and $O(n)$ edges in $P_{e}^{*}$, computation of $\tau$ takes time $O\left(n^{4}\right)$.

We remark that there is no guarantee that $\tau\left(e^{*}\right)$ and $\rho\left(e^{*}\right)$ have the same endpoints or indeed that $d\left(e^{*}, \tau\left(e^{*}\right)\right) \leq \kappa$. In Section 6 we guarantee both properties by in effect augmenting $\tau\left(e^{*}\right)$ to a polygonal chain using vertical edges connecting its endpoints to the endpoints of $\rho\left(e^{*}\right)$.

\section{Lifting Triangle Edges}

The rounding $\sigma(f)$ of a facet $f$ is defined from a lifting $l_{f}$ of the vertices, edges, and triangles of $T_{f}$. The definitions of $\sigma$ and $l_{f}$ appear in Section 6 below. This section defines an auxiliary lifting function $l_{f \Delta}(e)$ required to define $l_{f}(\Delta)$. For $e$ an edge of $\Delta \in T_{f}$, $l_{f \Delta}(e)$ is an edge over $e$; it will form part of the boundary of $l_{f}(\Delta)$.

The lifted edges $\left\{l_{f \Delta}(e): f \in F_{\Delta}\right\}$ must satisfy three properties: each edge $l_{f \Delta}(e)$ must be close to $f$; the edges must respect vertical order (i.e., $f \preceq f^{\prime}$ must imply $\left.l_{f \Delta}(e) \preceq l_{f^{\prime} \Delta}(e)\right)$, and no pair of lifted edges may cross. These properties are the main result of this section (Lemma 5.3). The definition of $l_{f \Delta}(e)$ and the proof of the properties are rather technical; on first reading it may be appropriate to skip to Section 6.

Section 5.1 below gives important technical tools for the rest of the section: "covering order" on the facets in $F_{\Delta}$ and the "snapping lemma." Covering order is used to order the choices of $\left\{l_{f \Delta}(e): f \in F_{\Delta}\right\}$. Suppose facet $f$ follows facet $f^{\prime}$ in covering order and $l_{f^{\prime} \Delta}(e)$ has been chosen to be close to $f^{\prime}$. If, say, $f \prec f^{\prime}$, then the snapping lemma guarantees that it is possible to choose $l_{f \Delta}(e)$ so that both $l_{f \Delta}(e) \preceq l_{f^{\prime} \Delta}(e)$ and $l_{f \Delta}(e)$ is close to $f$. In order to make the snapping lemma appropriately transitive, it is necessary to have a careful definition of what it means for an edge to be close to a facet ("the edge approximates the facet").

\subsection{The Order $\triangleleft$ and the Snapping Lemma}

Let edge $e$ of $T$ have endpoints $u$ and $v$. Define $R_{e}$ to be the convex hull of $\pi_{x y}\left(P_{e}^{*}\right)$, less the interior of $\operatorname{pixel}(u)$ and $\operatorname{pixel}(v)$, unioned with $\pi_{x y}\left(P_{e}^{*}\right)$. See Fig. 6. Notice that there are no intersections among the boundaries of $\left\{\pi_{x y}(f): f \in F_{\Delta}\right\}$ within $R_{e}$ except possibly at the endpoints of edges of $\pi_{x y}\left(P_{e}^{*}\right)$. Facet $f \in F_{e}$ covers $e$ if no edge in $P_{e}^{*}$ bounds $f$; it is easy to check that $R_{e} \subseteq \pi_{x y}(f)$. A facet $f$ covers facet $f^{\prime}$ at $e$ if $\pi_{x y}\left(f^{\prime}\right) \cap R_{e} \subseteq$ $\pi_{x y}(f) \cap R_{e}$. For any two facets $f, f^{\prime} \in F_{e}$, either $f$ covers $f^{\prime}$ at $e$, or $f^{\prime}$ covers $f$ at $e$.

Suppose that $e$ is an edge of triangle $\Delta$ of $T$. The covering order $\triangleleft$ on the facets in $F_{\Delta}$ is any total order so that $f \triangleleft f^{\prime}$ implies $f^{\prime}$ covers $f$ at $e$. (The order depends on both $e$ and $\Delta$, but to keep the notation simple we do not make this dependence explicit.) The order $\triangleleft$ can be described as follows. Assume that $\Delta$ lies to the left of the $e$, directed from endpoint 


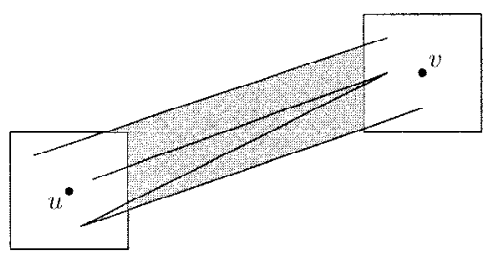

Fig. 6. $R_{e}$ is the shaded region plus the portion of the edges inside $\operatorname{pixel}(u)$ and $\operatorname{pixel}(v)$.

$u$ to endpoint $v$; direct all edges in $P_{e}^{*}$ from $\operatorname{pixel}(u)$ to $\operatorname{pixel}(v)$. If facets $f_{0}, f_{1} \in F_{\Delta}$ have bounding edges $e_{0}^{*}, e_{1}^{*} \in P_{e}^{*}$, then $f_{0} \triangleleft f_{1}$ if $e_{0}^{*}$ is to the left of $e_{1}^{*}$; all facets covering $e$ appear at the end of the order $\triangleleft$, and are ordered arbitrarily among themselves.

We remark that there may be facets in $F_{e}$ that are not in $F_{\Delta}$ for either of the two possible triangles $\Delta$ incident to $e$; these facets do not appear in the covering order. Such facets necessarily have two edges in $P_{e}^{*}$, both of which project and snap-round to $e$. Such edges (and edges in $P_{e}^{*}$ not incident to any facet) are important for the definition of $\sigma(f)$ and are discussed in Section 6.3 below.

For a set $S \subset \mathbb{R}^{3}$, let $V(S)$ be all points on all vertical lines through $S$. Let $f_{A}$ be a facet of $P$, let $e$ be an edge of $T$ with endpoints $u$ and $v$, and let $A$ be an edge over $e$. Edge $A$ approximates $f_{A}$ at $e$ if $d\left(A_{u}, f_{A} \cap V\left(R_{e}\right)\right)<\kappa$ and $d\left(A_{v}, f_{A} \cap V\left(R_{e}\right)\right)<\kappa$. Clearly, if $A$ approximates $f_{A}$ at $e$, then, by Proposition 3.1, $d\left(A, f_{A}\right)<\kappa$. Also, if $e^{*} \in P_{e}^{*}$ is a boundary edge of face $f$, then $d\left(\tau\left(e^{*}\right), e^{*}\right)<\kappa$ by Lemma 4.3, and $\tau\left(e^{*}\right)$ approximates $f$ at $e$.

Lemma 5.1 (Snapping Lemma). Let edge e bound triangle $\Delta$ of T. Suppose $f_{A}, f_{B}, f_{C}$ $\in F_{\Delta}$ with $f_{A} \succeq f_{C} \succeq f_{B}, A, B, C$ are edges over e approximating $f_{A}, f_{B}, f_{C}$, respectively, $A \succeq B$, and $f_{C}$ covers $f_{A}$ and $f_{B}$. Then $\operatorname{snap}(C,[A, B])$ also approximates $f_{C}$ ate.

Proof. We claim $\max (B, C)$ approximates $f_{C}$ at $e$; a similar result holds for min, from which the lemma follows. Let $u$ be an endpoint of $e$. We show $d\left(\max \left(B_{u}, C_{u}\right), f_{C} \cap\right.$ $\left.V\left(R_{e}\right)\right)<\kappa$. If $C_{u} \succeq B_{u}$, there is nothing to prove, so suppose $C_{u} \prec B_{u}$.

Let $T_{B}$ and $T_{C}$ be the cubes of sidelength $2 \kappa$ centered at $B_{u}$ and $C_{u}$, respectively, and $T=V\left(T_{B}\right)$ (clearly also $T=V\left(T_{C}\right)$ ). See Fig. 7 .

Since $B$ approximates $f_{B}$, there is a point $b \in f_{B} \cap V\left(R_{e}\right) \cap T_{B}$. Since $f_{C} \succeq f_{B}$ and $f_{C}$ covers $f_{B}$ at $e$, there is a point $c \in f_{C}$ with $c \succeq b$; clearly, $c \in T$. Since $C$ approximates $f_{C}$, there is a point $c^{\prime} \in f_{C} \cap V\left(R_{e}\right) \cap T_{C}$. Since $f_{C} \cap V\left(R_{e}\right) \cap T$ is path-connected, there is a path in $f_{C} \cap V\left(R_{e}\right) \cap T$ from $c$ to $c^{\prime}$. Since $c$ is above the bottom facet of $T_{B}, c^{\prime}$ is below the top facet of $T_{C}$, and $T_{B} \succeq T_{C}$, some point of the path meets $T_{B}$. Hence $d\left(B_{u}, f_{C}\right)<\kappa$.

\subsection{Default Edges}

Let $e$ be an edge of $T_{f}$ with endpoints $u$ and $v$ and with some triangle incident. We define the default lifting of edge e for facet $f, c_{f}(e)$, which is to be used in the absence of other 


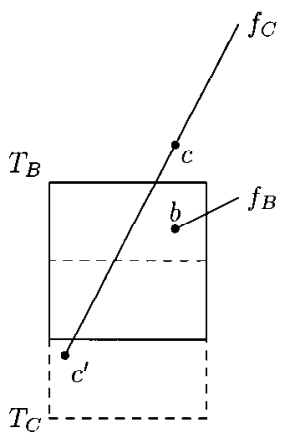

Fig. 7. Proof of Lemma 5.1, side view. $T_{B}$ is a solid square, $T_{C}$ is dashed.

constraints. If $e$ is a boundary edge of $T_{f}$, then there is a unique edge $e^{*} \in P_{e}^{*}$ bounding facet $f$, and we simply define $c_{f}(e)=\tau\left(e^{*}\right)$.

The definition of $c_{f}(e)$ is more complex if $e$ is an interior edge of $T_{f}$. Clearly, $f$ covers $e$ and no edge in $P_{e}^{*}$ meets $f$.

Define $\operatorname{low}_{f}(e)$ to be the edge $\hat{u} \hat{v}$, where $\hat{u}$ is the center of the lowest voxel $X$ in $\operatorname{column}(u)$ so that $X \cap f \cap V\left(R_{e}\right)$ is not empty, and similarly for $\hat{v}$. A pair of distinct edges $\left(a^{*}, b^{*}\right)$ in $P_{e}^{*}$ is a bracketing pair if $a^{*} \succ f$ and $f \succ b^{*}, \tau\left(a^{*}\right) \succeq \tau\left(b^{*}\right)$ and no edge $\tau\left(d^{*}\right), d^{*} \in P_{e}^{*}$, lies between $\tau\left(a^{*}\right)$ and $\tau\left(b^{*}\right)$ (possibly $\tau\left(a^{*}\right)=\tau\left(b^{*}\right)$ ). The existence of a bracketing pair can be seen by indexing the edges of $P_{e}^{*}=\left\{e_{0}^{*}, \ldots, e_{k}^{*}\right\}$ so that $\tau\left(e_{0}^{*}\right) \succeq \tau\left(e_{1}^{*}\right) \succeq \cdots \succeq \tau\left(e_{k}^{*}\right)$. Either $f \succ e_{0}^{*}$, and the pair ( $\left.\top, e_{0}^{*}\right)$ suffices (with the definition $\tau(\top)=\top$ ); or $e_{k}^{*} \succ f$, and the pair $\left(e_{k}^{*}, \perp\right)$ suffices; or there is $i$ so that an $e_{i} \succ f$ and $f \succ e_{i+1}$, and the pair $\left(e_{i+1}, e_{i}\right)$ suffices. It is possible that there are several bracketing pairs. Define

$$
c_{f}(e)=\operatorname{snap}\left(\operatorname{low}_{f}(e),\left[\tau\left(a^{*}\right), \tau\left(b^{*}\right)\right]\right),
$$

where $\left(a^{*}, b^{*}\right)$ is a bracketing pair chosen so that $\left(\tau\left(a^{*}\right), \tau\left(b^{*}\right)\right)$ is minimal in $\prec$ among bracketing pairs. Set $C_{e}=\left\{c_{f}(e): f \in F_{\Delta}\right\}$.

Lemma 5.2. Let $f$ be a facet of $P$ and let e be an edge of $T_{f}$.

(1) $c_{f}(e)$ approximates $f$ at $e$.

(2) If $f, f^{\prime}$ cover $e$ and $f \preceq f^{\prime}$, then $c_{f}(e) \preceq c_{f^{\prime}}(e)$.

(3) $\tau\left(P_{e}^{*}\right) \cup C_{e}$ is noncrossing.

Proof. (1) If $e$ is a bounding edge of $T_{f}$, then the claim is immediate. Otherwise $e$ is an interior edge of $T_{f}$ and

$$
c_{f}(e)=\operatorname{snap}\left(\operatorname{low}_{f}(e),\left[\tau\left(a^{*}\right), \tau\left(b^{*}\right)\right]\right),
$$

for some bracketing pair $\left(a^{*}, b^{*}\right)$. Let $a^{*}$ and $b^{*}$ be incident to faces $f_{a}$ and $f_{b}$, respectively. It is easy to check that $\operatorname{low}_{f}(e)$ approximates $f$. Clearly, $\tau\left(a^{*}\right)$ approximates $f_{a}$, $\tau\left(b^{*}\right)$ approximates $f_{b}$, and $f$ covers $f_{a}$ and $f_{b}$ (since $e$ is not a bounding edge of $T_{f}$ ). Part (1) is thus immediate from Lemma 5.1. 
(2) We have $R_{e} \subseteq \pi_{x y}(f), R_{e} \subseteq \pi_{x y}\left(f^{\prime}\right)$, and $f \prec f^{\prime}$, so $\operatorname{low}_{f}(e) \preceq \operatorname{low}_{f^{\prime}}(e)$. Let $a_{f}^{*}, b_{f}^{*}$ and $a_{f^{\prime}}^{*}, b_{f^{\prime}}^{*}$ be bracketing pairs for $f$ and $f^{\prime}$, respectively. We claim $\tau\left(a_{f}^{*}\right) \preceq \tau\left(a_{f^{\prime}}^{*}\right)$ and $\tau\left(b_{f}^{*}\right) \preceq \tau\left(b_{f^{\prime}}^{*}\right)$, from which $c_{f}(e) \preceq c_{f^{\prime}}(e)$ follows easily. Clearly, $\tau\left(a_{f}^{*}\right) \succeq \tau\left(b_{f}^{*}\right)$. It cannot be that $\tau\left(b_{f}^{*}\right) \succ \tau\left(a_{f^{\prime}}^{*}\right)$, for $a_{f^{\prime}}^{*} \succ f^{\prime} \succeq f$ and $f$ would have a bracketing pair below $\left(a_{f}^{*}, b_{f}^{*}\right)$, contradicting minimality. No edge of $\tau\left(P_{e}^{*}\right)$ lies between $\tau\left(a_{f}^{*}\right)$ and $\tau\left(b_{f}^{*}\right)$, so it must be that $\tau\left(a_{f^{\prime}}^{*}\right) \succeq \tau\left(a_{f}^{*}\right)$. Similarly, $\tau\left(b_{f^{\prime}}^{*}\right) \succeq \tau\left(b_{f}^{*}\right)$.

(3) By the definition of "snap," no edge $c_{f}(e)$ crosses an edge of $\tau\left(P_{e}^{*}\right)$. Also, clearly, if $f$ and $f^{\prime}$ have distinct bracketing pairs, then $c_{f}(e)$ and $c_{f^{\prime}}(e)$ do not cross. If $f$ and $f^{\prime}$ have the same bracketing pair, then $c_{f}(e)$ and $c_{f^{\prime}}(e)$ do not cross because $\operatorname{low}_{f}(e)$ and $\operatorname{low}_{f^{\prime}}(e)$ do not cross.

\subsection{Lifting Triangle Edges}

Let $e$ be an edge of triangle $\Delta$ of $T$. For facets $f \in F_{\Delta}$ in the order $\triangleleft$, simultaneously and inductively define $a_{f \Delta}(e)$ (the constraint from above), $b_{f \Delta}(e)$ (the constraint from below), and $l_{f \Delta}(e)$ (the lifting of edge e of $\Delta$ in $f$ ), as follows:

$$
\begin{aligned}
a_{f \Delta}(e) & =\min \left\{l_{f^{\prime} \Delta}(e): f^{\prime} \triangleleft f \text { and } f^{\prime} \succ f\right\}, \\
b_{f \Delta}(e) & =\max \left\{l_{f^{\prime} \Delta}(e): f^{\prime} \triangleleft f \text { and } f^{\prime} \prec f\right\}, \\
l_{f \Delta}(e) & =\operatorname{snap}\left(c_{f}(e),\left[a_{f \Delta}(e), b_{f \Delta}(e)\right]\right) .
\end{aligned}
$$

We have $a_{f \Delta}(e) \succeq b_{f \Delta}(e)$ by Lemma 5.3(1) below.

The definition is illustrated schematically in Fig. 8. For this configuration, we have

$$
\begin{aligned}
& l_{f_{0} \Delta}(e)=\operatorname{snap}\left(\tau\left(e_{0}^{*}\right),[\top, \perp]\right)=\tau\left(e_{0}^{*}\right), \\
& l_{f_{1} \Delta}(e)=\operatorname{snap}\left(\tau\left(e_{1}^{*}\right),\left[\top, l_{f_{0} \Delta}(e)\right]\right)=\tau\left(e_{0}^{*}\right), \\
& l_{f_{2} \Delta}(e)=\operatorname{snap}\left(\tau\left(e_{2}^{*}\right),\left[l_{f_{0} \Delta}(e), \perp\right]\right)=\tau\left(e_{0}^{*}\right), \\
& l_{f_{3} \Delta}(e)=\operatorname{snap}\left(c_{f_{3}}(e),\left[l_{f_{0} \Delta}(e), l_{f_{2} \Delta}(e)\right]\right)=\tau\left(e_{0}^{*}\right) .
\end{aligned}
$$

$F_{\Delta^{\prime}}$ contains only $f_{3}$, and $l_{f_{3} \Delta^{\prime}}(e)=c_{f_{3}}(e)$.

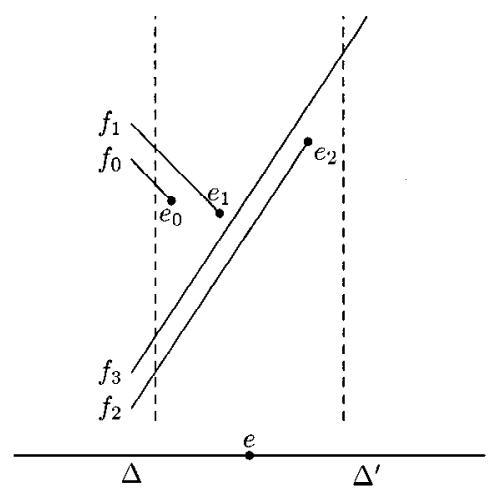

Fig. 8. Definition of $l_{f \Delta}(e)$, side view. $\Delta$ is incident to the left and $\Delta^{\prime}$ to the right. Vertical dotted lines outline the area that projects and snap-rounds to $e$. 
Lemma 5.3. Let $e$ be an edge of triangle $\Delta$ of $T$ with edge e, and let $f, f^{\prime} \in F_{\Delta}$.

(1) $a_{f \Delta}(e) \succeq b_{f \Delta}(e)$.

(2) If $f \preceq f^{\prime}$, then $l_{f \Delta}(e) \preceq l_{f^{\prime} \Delta}(e)$.

(3) $l_{f \Delta}(e)$ approximates $f$ at $e$.

(4) $l_{f \Delta}(e) \in \tau\left(P_{e}^{*}\right) \cup C_{e}$.

Proof. (1), (2) We prove both simultaneously by induction on $\triangleleft$. If $a_{f \Delta}(e)=\top$ or $b_{f \Delta}(e)=\perp, a_{f \Delta}(e) \succeq b_{f \Delta}(e)$ is immediate. Otherwise $a_{f \Delta}(e)=l_{f_{0} \Delta}(e)$ and $b_{f \Delta}(e)=$ $l_{f_{1} \Delta}(e)$ for some facets $f_{0} \succeq f \succeq f_{1}$, so by induction hypothesis $a_{f \Delta}(e) \succeq b_{f \Delta}(e)$. For (2), suppose $f \preceq f^{\prime}$; without loss of generality assume $f^{\prime} \triangleleft f$. Then by definition $l_{f^{\prime} \Delta}(e) \succeq a_{f \Delta}(e) \succeq l_{f \Delta}(e)$.

(3) Since $l_{f \Delta}(e)$ is defined in the order $\triangleleft$, the claim follows from an easy induction using Lemma 5.1.

(4) By Lemma 5.2, $\tau\left(P_{e}^{*}\right) \cup C_{e}$ is noncrossing, so the "snap" in the definition of $l_{f \Delta}(e)$ results in an element of $\tau\left(P_{e}^{*}\right) \cup C_{e}$.

For $e$ an edge of $T$, define $L_{e}=C_{e} \cup\left\{l_{f \Delta}(e): \Delta\right.$ is incident to $e$ and $\left.f \in P_{\Delta}\right\} . L_{e}$ is all of the edges that have been defined over $e$.

Corollary 5.4. $L_{e}$ is noncrossing.

Proof. Lemmas 5.3(4) and 5.2(3), using the fact that the construction of $C_{e}$ does not depend upon the choice of triangle incident to $e$.

\section{The Subdivision $Q$}

In this section we define the subdivision $Q$ and the embedding $\sigma$ of $P$ into $Q$ required by Theorem 2.1. This section also contains the definitions of $l_{f}$ for the vertices, edges, and triangles of $T$.

\subsection{Vertices and Edges}

Let $v^{*}$ be a vertex of $P^{*}$. Define $\sigma\left(v^{*}\right)=\rho\left(v^{*}\right)$ (recall $\rho\left(v^{*}\right)$ is the center of the voxel containing $v^{*}$ ).

Let $v$ be a vertex of $T$. The vertical carrier $V C(v)$ is the vertical chain of edges through $\rho\left(P_{v}^{*}\right)$, i.e. all edges connecting two vertices of $\rho\left(P_{v}^{*}\right)$ that are adjacent in vertical order.

Let $e^{*} \in P_{e}^{*}$, where edge $e$ in $T$ has endpoints $u$ and $v$. Define $\sigma\left(e^{*}\right)$ to be the subdivision consisting of $\tau\left(e^{*}\right)$, the subchain of $V C(u)$ connecting $\tau\left(e^{*}\right)_{u}$ to $\rho\left(e^{*}\right)_{u}$ and the subchain of $V C(v)$ connecting $\tau\left(e^{*}\right)_{v}$ to $\rho\left(e^{*}\right)_{v}$. Extend $\sigma$ to edges $e$ of $P$ :

$$
\sigma(e)=\bigcup_{e^{*} \in P^{*}, e^{*} \subseteq e} \sigma\left(e^{*}\right) .
$$

Clearly, $\sigma(e)$ is a subdivision. 
Lemma 6.1. If $w, w^{\prime}$ are vertices or edges of $P$ and $w \preceq w^{\prime}$, then $\sigma(w) \preceq \sigma\left(w^{\prime}\right)$.

Proof. The claim is immediate for two vertices. Suppose $w$ is a vertex and $w^{\prime}$ is an edge; the symmetric case is similar. Then $\sigma\left(w^{\prime}\right)$ contains a vertical chain of edges from the center of the first voxel in column $(w)$ met by $w^{\prime}$ to the center of the last such voxel. Since $w \prec w^{\prime}, \rho(w)$ is below or on the chain, and $\sigma(w) \preceq \sigma\left(w^{\prime}\right)$. The case of two edges is similar.

Let $e$ be an edge of $T$ with endpoints $u$ and $v$. Split each edge in $L(e)$ at its midpoint. Split each edge at its midpoint. These edges together with $V C(u)$ and $V C(v)$ form a planar graph (in the plane through $V C(u)$ and $V C(u)$ ). The vertical carrier $V C(e)$ is an arbitrary triangulation of this graph.

\subsection{Triangles}

Let $\Delta$ be a triangle of $T_{f}$ with vertices $a, b, c$. Consider the edges $l_{f \Delta}(a b), l_{f \Delta}(a c)$, $l_{f \Delta}(b c)$. There is no guarantee that these edges are pairwise incident (of course both $l_{f \Delta}(a b)$ and $l_{f \Delta}(a c)$ are incident to vertices over $a$, and similarly for the other pairs). We form a (three-dimensional) polygon from $l_{f \Delta}(a b), l_{f \Delta}(a c), l_{f \Delta}(b c)$ by adding the vertical subchain of $L(a)$ connecting $l_{f \Delta}(a b)_{a}$ to $l_{f \Delta}(a c)_{a}$ (if they are not equal) and similarly for the $b$ and $c$ endpoints. The lifting of $\Delta$ for facet $f, l_{f}(\Delta)$, is a triangulation of this polygon, described as follows.

Split edges $l_{f \Delta}(a b), l_{f \Delta}(a c), l_{f \Delta}(b c)$ at their respective midpoints $m_{a b}, m_{a c}, m_{b c}$, and add the three edges connecting midpoints. This forms a central triangle $m_{a b} m_{a c} m_{b c}$ and three polygons, where, for example, the a-polygon (of $f$ ) consists of edge $m_{a b} m_{a c}$, the two subedges of $l_{f \Delta}(a b)$ and $l_{f \Delta}(a c)$ with endpoints over $a$, and possibly a vertical chain over $a$. See Fig. 9.

For points $p, q \in \mathbb{R}^{3}$ and $\alpha \in \mathbb{R}$, let $\alpha[p, q]$ be the point $(1-\alpha) p+\alpha q$, i.e., the point a fraction $\alpha$ of the way from $p$ to $q$.

The $a$-index of $f$ is the number of distinct pairs $\left(l_{f^{\prime} \Delta}(a b), l_{f^{\prime} \Delta}(a c)\right)$, where $f^{\prime} \succeq f$. Let $\alpha_{f}=i / 2^{\left\lceil\log _{2} n\right\rceil}$, where $i$ is the $a$-index of $f$; clearly, $0<\alpha_{f}<1$. First assume

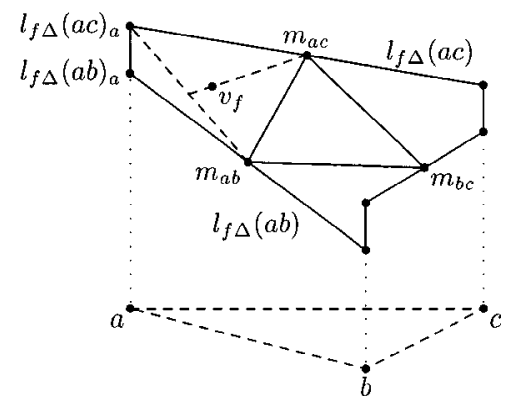

Fig. 9. Definition of $v_{f}$ for triangle $\Delta$ of $T_{f}$ with vertices $a, b, c$. 
$l_{f \Delta}(a c)_{a} \succeq l_{f \Delta}(a b)_{a}$. Set $v_{f}=\alpha_{f}\left[\frac{1}{2}\left[l_{f \Delta}(a c)_{a}, m_{a b}\right], m_{a c}\right]$. See Fig. 9. Triangulate the $a$-polygon of $f$ with $v_{f}$, i.e., connect $v_{f}$ to $m_{a c}, m_{a b}$, and any vertex on the chain from $l_{f \Delta}(a b)_{a}$ to $l_{f \Delta}(a c)_{a}$. If $l_{f \Delta}(a b)_{a} \succ l_{f \Delta}(a c)_{a}$, the construction is similar, with $m_{a b}$ and $m_{a c}$ interchanged and $l_{f \Delta}(a b)_{a}$ substituting for $l_{f \Delta}(a c)_{a}$. The other two polygons are triangulated in a similar fashion.

Lemma 6.2. Let $\Delta$ be a triangle of $T$ and $f, f^{\prime} \in F_{\Delta}$.

(1) $d\left(l_{f}(\Delta), f\right)<\kappa$.

(2) If $f \preceq f^{\prime}$, then $l_{f}(\Delta) \preceq l_{f^{\prime}}(\Delta)$.

(3) Every vertex coordinate of $l_{f}(\Delta)$ is an integral multiple of $1 / 2^{\left\lceil\log _{2} n\right\rceil+2}$.

(4) $l_{f}(\Delta)$ has $O(n)$ cells.

Proof. (1) Let $\Delta$ have vertices $a, b, c$. Every vertex of $l_{f}(\Delta)$ is within the convex hull of $\left\{l_{f \Delta}(a b), l_{f \Delta}(a c), l_{f \Delta}(b c)\right\}$. The claim follows using Lemma 5.3(1) and Proposition 3.1.

(2) We can assume that $l_{f \Delta}(a c)_{a} \succeq l_{f \Delta}(a b)_{a}$. Using Lemma 5.3(2), we must have

$$
l_{f^{\prime} \Delta}(a c)_{a} \succeq l_{f \Delta}(a c)_{a} \quad \text { and } \quad l_{f^{\prime} \Delta}(a b)_{a} \succeq l_{f \Delta}(a b)_{a} .
$$

If $l_{f^{\prime} \Delta}(a b)_{a} \succ l_{f \Delta}(a c)_{a}$, then the result is immediate, since the convex hull of $\left\{l_{f \Delta}(a b)\right.$, $\left.l_{f \Delta}(a c)\right\}$ and the convex hull of $\left\{l_{f^{\prime} \Delta}(a b), l_{f^{\prime} \Delta}(a c)\right\}$ have disjoint interiors. Hence we can assume that

$$
l_{f^{\prime} \Delta}(a c)_{a} \succeq l_{f \Delta}(a c)_{a} \succeq l_{f^{\prime} \Delta}(a b)_{a} \succeq l_{f \Delta}(a b)_{a} .
$$

Let $i_{f}$ and $i_{f^{\prime}}$ be the $a$-indices of $f$ and $f^{\prime}$, respectively. If $i_{f}=i_{f^{\prime}}$, then the $a$-polygons for $f$ and $f^{\prime}$ are identical. Otherwise, $i_{f}>i_{f^{\prime}}$ since $f \preceq f^{\prime}$. Let $s$ be the edge connecting $v_{f^{\prime}}$ to the midpoint of $l_{f^{\prime} \Delta}(a c)$; clearly, we have $\pi_{x y}\left(v_{f}\right) \in \pi_{x y}(s)$ since $\alpha_{f}>\alpha_{f^{\prime}}$. Furthermore, we have $v_{f} \prec s$, since $l_{f \Delta}(a b) \preceq l_{f^{\prime} \Delta}(a b)$ and $l_{f \Delta}(a c) \preceq l_{f^{\prime} \Delta}(a c)$ with inequality holding in at least one case. $l_{f}(\Delta) \preceq l_{f^{\prime}}(\Delta)$ follows easily.

(3), (4) Immediate.

\subsection{Vertical Ordering}

It is tempting to define $\sigma(f)=\bigcup_{\Delta \in T_{f}} l_{f}(\Delta)$. By Lemma 6.2(2), this definition would preserve or collapse vertical order (in the sense of Theorem 2.1) among lifted triangles. However, order would not necessarily be preserved between lifted triangles and rounded edges or vertices. To see why, let $e$ be an edge of $T_{f}$ with two triangles $\Delta$ and $\Delta^{\prime}$ incident. It is possible that there is an isolated edge $e^{*} \in P_{e}^{*}$ with no facet of $P$ incident (or similarly an edge $e^{*}$ of facet $f \in P_{e} \backslash\left(P_{\Delta} \cup P_{\Delta^{\prime}}\right)$, i.e., $f$ has two edges projecting and snaprounding to $e$ ). It is furthermore conceivable that $e^{*} \prec f$ but that both $l_{f \Delta}(e) \prec \tau\left(e^{*}\right)$ and $l_{f \Delta^{\prime}}(e) \prec \tau\left(e^{*}\right)$. With the tempting definition above, the vertical order between $e^{*}$ and $f$ would not be preserved by rounding. The solution, given below, is specially to define $l_{f}(e)$ as a (triangulated) vertical polygon, and include $l_{f}(e)$ in $\sigma(f)$. Similarly, for a vertex $v \in T, l_{f}(v)$ is defined as a vertical chain. We remark that in consequence $\sigma(f)$ may not be a 2-manifold; it may include vertical chains and polygons over vertices and edges of $T$. 
For a facet $f$, let $E_{f}(v)$ be all endpoints of edges $\left\{l_{f \Delta}(e): e, \Delta \in T\right\}$ over $v$ (clearly, edge $e$ must be incident to $v$ and $\Delta$ ). Define

$$
\begin{aligned}
& a_{f}(v)=\min \left(\left\{\sigma\left(v^{*}\right): v^{*} \in P_{v}^{*} \text { and } v^{*} \succeq f\right\} \cup E_{f}(v)\right), \\
& b_{f}(v)=\max \left(\left\{\sigma\left(v^{*}\right): v^{*} \in P_{v}^{*} \text { and } v^{*} \preceq f\right\} \cup E_{f}(v)\right) .
\end{aligned}
$$

Informally, $a_{f}(v)$ is the lowest snap-rounding over $v$ of a vertex on or above $f$; similarly, $B_{f}(v)$ is the highest snap-rounding over $v$ of a vertex on or below $f$. Easily $a_{f}(v) \preceq$ $b_{f}(v)$. The lifting of vertex $v$ for facet $f, l_{f}(v)$, is the subchain of $V C(v)$ connecting $a_{f}(v)$ and $b_{f}(v)$.

For a facet $f$ and an edge $e$ of $T_{f}$, let $E_{f}(e)=\left\{l_{f \Delta}(e): \Delta\right.$ incident to $e$ in $\left.T_{f}\right\}$. Clearly, there are are zero, one, or two edges in $E_{f}(e)$ as there are zero, one, or two triangles incident to $e$ in $T_{f}$. Define

$$
\begin{aligned}
& a_{f}(e)=\min \left(\left\{\tau\left(e^{*}\right): e^{*} \in P_{e}^{*} \text { and } e^{*} \succeq f\right\} \cup E_{f}(e)\right), \\
& b_{f}(e)=\max \left(\left\{\tau\left(e^{*}\right): e^{*} \in P_{e}^{*} \text { and } e^{*} \preceq f\right\} \cup E_{f}(e)\right) .
\end{aligned}
$$

Notice that if $E_{f}(e)$ is empty, then there must be some edge of $e^{*} \in P_{e}^{*}$ incident to $f$, so $a_{f}(e)$ and $b_{f}(e)$ are distinct from $\perp$ and $T$, respectively. The lifting of edge e for facet $f, l_{f}(e)$, is all edges and vertices $w$ of $V C(e)$ satisfying $b_{f}(e) \succeq w$ and $w \succeq a_{f}(e)$.

Lemma 6.3. Suppose $w$ is a vertex or edge of $T, w^{*} \in P_{w}^{*}$, and $f$ is a facet of $P$. Then $w^{*} \preceq f$ implies $\sigma\left(w^{*}\right) \preceq l_{f}(w)$ and $w^{*} \succeq f$ implies $\sigma\left(w^{*}\right) \succeq l_{f}(w)$.

Proof. By construction.

Lemma 6.4. Let $f$ be a facet of $P$ and let $w$ be a vertex or edge of $T_{f}$. Then $d\left(l_{f}(w), f\right)$ $\leq \kappa$.

Proof. Similar to the proof of Lemma 5.1.

For each facet $f$ of $P$, define

$$
\sigma(f)=\bigcup_{w \in T_{f}} l_{f}(w)
$$

where $w$ varies over vertices, edges, and triangles. It is easy to check that $\sigma(f)$ is a subdivision.

Lemma 6.5. If $f, f^{\prime}$ are cells of $P$ and $f \prec f^{\prime}$, then $\sigma(f) \preceq \sigma\left(f^{\prime}\right)$.

Proof. The lemma follows from Lemmas 6.1 and 6.3 if one of $f$ and $f^{\prime}$ is a vertex or edge. So suppose both are facets. For each triangle $\Delta$ in both $T_{f}$ and $T_{f^{\prime}}, l_{f}(\Delta) \preceq$ $l_{f^{\prime}}(\Delta)$ by Lemma 6.2. Suppose $e$ is an edge in both $T_{f}$ and $T_{f^{\prime}}$. If there is a triangle $\Delta$ in both $T_{f}$ and $T_{f^{\prime}}$ incident to $e$, Lemma 6.2 again implies $l_{f}(e) \preceq l_{f^{\prime}}(e)$. Otherwise, up to symmetry, there is an edge $e^{*} \in P_{e}^{*}$ bounding $f$ with $e^{*} \preceq f^{\prime}$, so, by 
Lemma 6.3, $\sigma\left(e^{*}\right) \preceq l_{f^{\prime}}(e)$. Since $e^{*} \preceq f, \sigma\left(e^{*}\right) \subseteq l_{f}(e)$, and $l_{f}(e) \preceq l_{f^{\prime}}(e)$. A similar argument shows that if $v$ is a vertex in both $T_{f}$ and $T_{f^{\prime}}$, then $l_{f}(v) \preceq l_{f^{\prime}}(v)$. Hence $\sigma(f) \preceq \sigma\left(f^{\prime}\right)$.

\subsection{The Subdivision $Q$}

Let

$$
Q=\bigcup_{f} \sigma(f),
$$

where $f$ varies over all facets of $P$. It is easy to check that $Q$ is a subdivision and that $\sigma$ is an embedding of $P$ into $Q$.

Lemma 6.6. $Q$ has $O\left(n^{4}\right)$ cells and can be computed in time $O\left(n^{4}\right)$.

Proof. For each facet $f$ of $P, T_{f}$ has $O\left(n^{2}\right)$ triangles $\Delta$. By Lemma $6.2, l_{f}(\Delta)$ has $O(n)$ cells. Hence $\sigma(f)$ has $O\left(n^{3}\right)$ cells, for a total of $O\left(n^{4}\right)$ over all facets of $f . Q$ can easily be computed in the same time.

\section{Hausdorff Distance}

It is immediate from Lemmas 6.2 and 6.4 that $d(\sigma(f), f) \leq \kappa$. In this section we show that $d(f, \sigma(f)) \leq \kappa$, implying $d_{\mathrm{H}}(\sigma(f), f) \leq \kappa$ and completing the proof of Theorem 2.1. This part of the proof has a topological flavor.

To illustrate the proof, we first give a one-dimensional analogue. Suppose we have a line segment $E=e_{0} e_{k}$ and a polygonal chain $C=c_{0} c_{1}, c_{1} c_{2}, \ldots, c_{k-1} c_{k}$ satisfying

1. $d\left(c_{i}, E\right)<\kappa$, and

2. $d\left(e_{0}, c_{0}\right)<\kappa$ and $d\left(e_{k}, c_{k}\right)<\kappa$.

See Fig. 10. Note that condition 1 immediately implies $d(C, E)<\kappa$ using Proposition 3.1; we wish to establish $d(E, C)<\kappa$ (which is clearly false without condition 2).

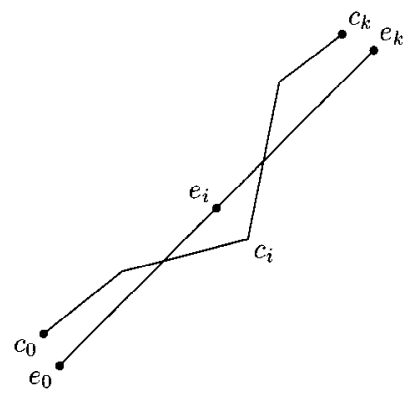

Fig. 10. $C$ is the chain $c_{0} c_{1}, \ldots, c_{k-1} c_{k}$; edge $E$ is $e_{0} e_{k}$. 
The proof is sketched as follows, using the same terminology as the two-dimensional case below. For $i=1, \ldots, k-1$, choose $e_{i}$ as the point of $E$ closest to $c_{i}$, then $d\left(c_{i}, e_{i}\right)<$ $\kappa$ for all $i=0, \ldots, k$. Let $A$ be a chain of $k$ (abstract) edges, $A=a_{0} a_{1}, \ldots, a_{k-1} a_{k}$. We can view $C$ and $E$ as embeddings $\varphi_{C}$ and $\varphi_{E}$ of $A$, i.e., $\varphi_{C}\left(a_{i}\right)=c_{i}$ and $\varphi_{E}\left(a_{i}\right)=e_{i}$. Then we have easily (compare Lemma 7.3)

(a) $\varphi_{E}(A) \subset E$ and $\varphi_{E}(A)$ covers $E$, i.e., $\varphi_{E}$ maps the endpoints of $A$ to the endpoints of $E$.

(b) Embeddings $\varphi_{E}$ and $\varphi_{C}$ are close, i.e., $d\left(\varphi_{C}\left(a_{i}\right), \varphi_{E}\left(a_{i}\right)\right)<\kappa$ for $i=0, \ldots, k$.

Item (a) implies that $E=\varphi_{E}(A)$ (compare Lemma 7.1) while (b) implies that $d_{\mathrm{H}}\left(\varphi_{E}(A)\right.$, $\left.\varphi_{C}(A)\right)<\kappa($ compare Lemma 7.2); these two assertions together yield the desired conclusion.

We now return to the two-dimensional case. An abstract triangle, $\Delta a b c$, is a cyclically ordered set of distinct abstract vertices $a, b, c$ (so $\Delta a b c=\Delta b c a=\Delta c a b \neq \Delta a c b$ ); $\Delta a b c$ has directed edges $a b, b c$, and $c a$. An abstract triangulation $A$ is a set of abstract triangles so that for each directed edge $a b$, there is a unique triangle with directed edge $b a$. An abstract triangulation $A$ has boundary $\partial A=\left\{a_{0} a_{1}, a_{1} a_{2}, \ldots, a_{k} a_{0}\right\}$ if there is a distinguished vertex $i$ (the point at infinity) so that $\Delta i a_{1} a_{0}, \ldots, \Delta i a_{k} a_{k-1}, \Delta i a_{0} a_{k}$ are exactly the triangles that have $i$ as a vertex. An embedding $\varphi$ of an abstract triangulation is a mapping from vertices (except the point at infinity) into $\mathbb{R}^{3}$. Embedding $\varphi$ extends to edges, triangles, and all of $A: \varphi(a b)=\varphi(a) \varphi(b), \varphi(\Delta a b c)$ is the convex hull of $\{\varphi(a), \varphi(b), \varphi(c)\}$, and $\varphi(A)=\bigcup_{\Delta \in A} \varphi(\Delta)$. Embedding $\varphi$ may map two vertices to the same point of $\mathbb{R}^{3}$, cause two triangles to intersect, etc.

Let $\varphi$ be an embedding of triangulation $A$ with boundary $\partial A$ and let $f$ be a facet of $P ; \varphi$ covers $f$ if $\varphi(v) \in f$ for all vertices $v \in A ; \varphi(\partial A)=\partial f$; and for distinct edges $e, e^{\prime}$ of $\partial A, \varphi(e)$ and $\varphi\left(e^{\prime}\right)$ have disjoint interiors.

Lemma 7.1. Let $\varphi$ be an embedding of A. If $\varphi$ covers $f$, then $f=\varphi(A)$.

Proof. Clearly, $\varphi(A) \subseteq f$. For the converse, let $p \in f$. Choose a directed line $l$ through $p$ in the plane of $f$ so that $l$ avoids all vertices of $\varphi(A)$. Choose an arbitrary orientation of the plane through $f$. Consider the directed graph whose nodes are the triangles $\Delta$ of $A$ so that $\varphi(\Delta) \cap l \neq \emptyset$ and whose arcs are directed from $\Delta a b c$ to $\Delta a c d$ if $a$ lies to the left of $l$ and $c$ to its right. Clearly, each triangle has indegree at most one and outdegree at most one. There is a unique edge in $\partial A$ that contains the first point of $l \cap f$; hence there is a unique triangle $\Delta_{0}$ of indegree 0 . Similarly, there is a unique triangle $\Delta_{k}$ of outdegree 0 . Hence there is a path of triangles $\Delta_{0}, \Delta_{1}, \ldots, \Delta_{k}$. Consider the edges $e_{i}=\varphi\left(\Delta_{i}\right) \cap l$. Consecutive edges share endpoints, so the union of the edges is $l \cap f$. Hence $p \in e_{i}$, some $i$, and $p \in \varphi\left(\Delta_{i}\right) \subseteq f$.

An embedding $\hat{\varphi}$ of $A$ is close to embedding $\varphi$ if, for all vertices $v$ of $A, d(\hat{\varphi}(v), \varphi(v))$ $<\kappa$.

Lemma 7.2. If embeddings $\varphi, \hat{\varphi}$ of $A$ are close, then $d_{\mathrm{H}}(\varphi(A), \hat{\varphi}(A))<\kappa$. 
Proof. Immediate using Proposition 3.1.

Lemma 7.3. For each facet $f$ of $P$, there is an abstract triangulation $A$ and close embeddings $\varphi, \hat{\varphi}$ so that $\hat{\varphi}$ covers $f$ and $\varphi(A) \subseteq \sigma(f)$.

Proof. We first assume that $T_{f}$ has at least one triangle and that every edge of $T_{f}$ is incident to a triangle. $A$ is obtained by pasting together various subtriangulations, using $T_{f}$ as a guide. For each triangle $\Delta$ in $T_{f}, A$ has an abstract copy of $l_{f}(\Delta)$, i.e., a set of abstract triangles with the same incidence structure as $l_{f}(\Delta) ; \varphi$ maps each abstract vertex to the corresponding vertex of $l_{f}(\Delta)$. A contains an abstract copy of $\sigma(\partial f)$, i.e., an abstract cycle formed from a copy of $\sigma(e)$ for each edge $e$ in $\partial f ; \varphi$ maps each abstract vertex to the corresponding vertex of $\sigma(\partial f)$. The boundary of $A$ is formed by an abstract copy of $\partial f ; \varphi$ maps each abstract vertex to the image under $\sigma$ of the corresponding vertex of $\partial f$. (Each edge in the boundary of $A$ forms a triangle with the point at infinity.)

The abstract copies are connected together as follows. For each internal edge $e$ between two triangles $\Delta$ and $\Delta^{\prime}$, the abstract copies of $l_{f \Delta}(e)$ and $l_{f \Delta^{\prime}}(e)$ are connected with intermediate abstract triangles (see Fig. 11). Similarly, $e$ is a boundary edge of $T_{f}$ and is incident to a triangle $\Delta$, the abstract copies of $\sigma(e)$ and $l_{f \Delta}(e)$ are connected by intermediate abstract triangles. For each vertex $v$ of $T_{f}$, the vertices of $A$ that are abstract copies of $v$ have been connected to form a cycle; this cycle is now triangulated. Finally, the cycles formed by abstract copies of $\partial f$ and $\sigma(\partial(f))$ are connected: each copy of a vertex $v \in \partial f$ is connected by an edge to the copy of $\sigma(v) \in \sigma(\partial(f))$, and each copy of an edge $e \in \partial f$ is connected by intermediate triangles to the copy of $\sigma(e) \in \sigma(\partial(f))$.

Clearly, we have $\varphi(A) \subseteq \sigma(f)$. Define $\hat{\varphi}$ on $\partial A$ by mapping the copy of a vertex $v \in \partial f$ to $v$; define $\hat{\varphi}$ elsewhere by mapping abstract vertex $u \in A$ to the closest point on $f$ to $\varphi(u)$. Clearly, $\hat{\varphi}$ covers $f$ and is close to $\varphi$.

If $T_{f}$ has an edge $e$ without incident triangles, the approach is similar, using an abstract copy of $l_{f}(e)$. The case that $T_{f}$ consists of a single vertex can be handled trivially.

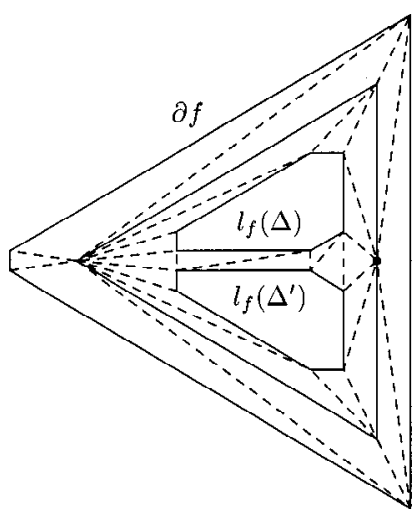

Fig. 11. Abstract triangulation $A$. Outer solid cycle is copy of $\partial f$; middle solid cycle is copy of $\sigma(\partial(f)$; inner polygons are copies of $l_{f}(\Delta)$ and $l_{f}\left(\Delta^{\prime}\right)$ (internal edges not shown). Dotted edges are connecting triangulation edges. 
Corollary 7.4. If $f$ is a facet of $P$, then $d_{\mathrm{H}}(f, \sigma(f))<\kappa$.

Proof. Immediate from Lemmas 7.1-7.3.

\section{Discussion}

It may be possible to improve the worst-case bounds given in Theorem 2.1. For example, the $O\left(n^{4}\right)$ bound on the size of $Q$ could be an artifact of vertical projection; perhaps an $O\left(n^{3}\right)$ bound could be obtained by using different projection directions in different places, each tuned to the local configuration. Obtaining a worst-case bound below $O\left(n^{3}\right)$ seems very challenging. It would be desirable to remove the extra $\left\lceil\log _{2} n\right\rceil+2$ bits needed for vertex coordinates; again, this may be an artifact of vertical projection. Finally, it would be desirable to guarantee that the rounded image of a facet at least was locally a 2-manifold.

A programmer would probably prefer a simple rounding algorithm, even at the expense of degraded worst-case bounds, as long as the typical-case bounds are reasonable. One reason that the rounding algorithm is complicated is the need to avoid edge crossings. Milenkovic [17] suggests rounding existing vertices to integer coordinates. If two rounded edges cross, then a vertex of intersection is added, with coordinates computed exactly. This would require a constant-factor increase in the bit-length of some vertex coordinates, and hence of some predicate evaluations. However, the maximum required bit-length is still bounded, and perhaps the increased-length calculations are relatively infrequent. Perhaps this approach can lead to a practical rounding algorithm.

\section{Appendix A. Proof of Lemma 4.2}

Proof. Let $e$ have endpoints $u$ and $u^{\prime}$, let $e^{*}$ have endpoints $E$ and $E^{\prime}$, and let $d^{*}$ have endpoints $D$ and $D^{\prime}$, where $D, E \in \operatorname{column}(u)$ and $D^{\prime}, E^{\prime} \in \operatorname{column}\left(u^{\prime}\right)$. By the definition of $P^{*}$, both $d^{*}$ and $e^{*}$ each intersect only a single voxel of column $(u)$ and a single voxel of column $\left(u^{\prime}\right)$. By clipping $d^{*}$ and $e^{*}$ slightly, we can assume that $D$ and $E$ lie on a bounding facet of column $(u)$ and $D^{\prime}$ and $E^{\prime}$ lie on a bounding facet of $\operatorname{column}\left(u^{\prime}\right)$.

We write, e.g., $D_{x}$ for the $x$-coordinate of $D$. Without loss of generality we can assume $D_{x}<D_{x}^{\prime}, D_{y}<D_{y}^{\prime}, D_{z}<D_{z}^{\prime}$, and since $\rho\left(d^{*}\right)$ and $\rho\left(e^{*}\right)$ cross, we can assume that $E_{z}>D_{z}$ and $E_{z}^{\prime}<D_{z}^{\prime}$. It cannot be that $u_{y}=u_{y}^{\prime}$, for then $D, D^{\prime}, E, E^{\prime}$ would lie on facets of column $(u)$ and column $\left(u^{\prime}\right)$ parallel to the $x z$-plane, and $\pi_{x z}\left(d^{*}\right)$ and $\pi_{x z}\left(e^{*}\right)$ would cross, which is impossible by the construction of $P^{*}$. Similarly, $u_{x} \neq u_{x}^{\prime}$, and we can assume that $u_{x}<u_{x}^{\prime}$ and $u_{y}<u_{y}^{\prime}$. Thus $D$ and $E$ must either lie on the $x z$-facet of column $(u)$, that is, the facet of column $(u)$ parallel to the $x z$-plane on the $+y$ side of column $(u)$, or the $y z$-facet on the $+x$ side. Similarly, $D^{\prime}$ and $E^{\prime}$ lie either on the $x z$-facet on the $-y$ side of column $\left(u^{\prime}\right)$ or the $y z$-facet on the $-x$ side.

The proof now splits into two rather different cases, depending on whether $E_{z}<E_{z}^{\prime}$ or $E_{z}>E_{z}^{\prime}$. 


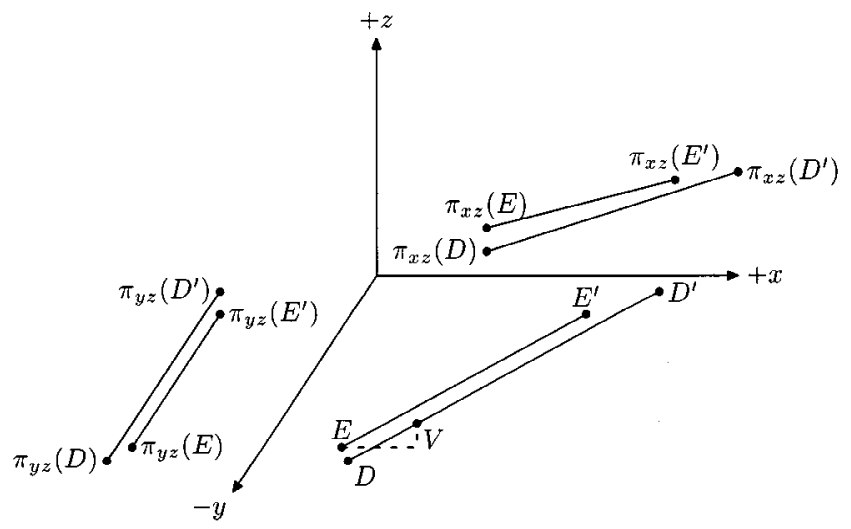

Fig. 12. Case 1 of the proof of Lemma 4.2.

Case 1: $E_{z}<E_{z}^{\prime}$. We have $D_{z}<E_{z}<E_{z}^{\prime}<D_{z}^{\prime}$. By the construction of $P^{*}, \pi_{x z}\left(d^{*}\right)$ and $\pi_{x z}\left(e^{*}\right)$ do not cross. We assume $\pi_{x z}\left(e^{*}\right) \succeq \pi_{x z}\left(d^{*}\right)$; the other case is symmetric, as will be evident momentarily. See Fig. 12. Since $\pi_{x z}\left(e^{*}\right) \succeq \pi_{x z}\left(d^{*}\right)$ and $D_{z}^{\prime}>E_{z}^{\prime}$, we must have $D_{x}^{\prime}>E_{x}^{\prime}$. Hence $D^{\prime}$ lies on the $x z$-facet of column $\left(u^{\prime}\right)$. The plane through the $x z$-facet intersects $e^{*}$ at some point with $z$-coordinate below $D_{z}^{\prime}$ (since this is true for all points of $\left.e^{*}\right)$, hence $\pi_{y z}\left(d^{*}\right) \succeq \pi_{y z}\left(e^{*}\right)$. By a similar argument, $D$ lies on the $y z$-facet of column $(u) . E$ and $E^{\prime}$ could be on either facet of column $(u)$ and column $\left(u^{\prime}\right)$, respectively. See also Fig. 2. (The case $\pi_{x z}\left(e^{*}\right) \preceq \pi_{x z}\left(d^{*}\right)$ would be symmetric, with $\pi_{y z}\left(d^{*}\right) \preceq \pi_{y z}\left(e^{*}\right)$.)

Assume that the angle $\alpha$ between the $y$-axis and the line through $\pi_{x y}\left(d^{*}\right)$ is at most $\pi / 4$; we show $d\left(\rho(E), d^{*}\right) \leq \kappa$. (The case $\alpha \in[\pi / 4, \pi / 2]$ implies $d\left(\rho\left(E^{\prime}\right), d^{*}\right) \leq \kappa$.) Let $V$ be the point of $d^{*}$ with the same $y$-coordinate as $E$; certainly $V_{z}>E_{z}$. We have $d\left(\pi_{x y}(E), \pi_{x y}(D)\right)<1$ and $d\left(\pi_{x y}(E), \pi_{x y}(V)\right)<1$ since $\left|E_{y}-D_{y}\right|<1$ and $\alpha<\pi / 4$. We also have $D_{z}<E_{z}<V_{z}$; let $W$ be the point on edge $D V$ with the same $z$-coordinate as $E$. By Proposition 3.1, $d\left(\pi_{x y}(E), \pi_{x y}(W)\right)<1$, thus $d(E, W)<1, d\left(E, d^{*}\right)<1$, and $d\left(\rho(E), d^{*}\right)<\frac{3}{2}=\kappa$.

Case 2: $E_{z}^{\prime}<E_{z}$. We cannot have $E_{x}^{\prime}=D_{x}^{\prime}$, else $\pi_{x y}\left(d^{*}\right)$ and $\pi_{x y}\left(e^{*}\right)$ would cross. Suppose $E_{x}^{\prime}>D_{x}^{\prime}$. Then we have $E^{\prime}$ on the $x z$-facet of column $\left(u^{\prime}\right)$. Furthermore, $E_{z}>E_{z}^{\prime}$ and $\pi_{x z}\left(e^{*}\right) \succeq \pi_{x z}\left(d^{*}\right)$, since $\pi_{x z}\left(d^{*}\right)$ and $\pi_{x z}\left(e^{*}\right)$ do not cross. We cannot also have $D^{\prime}$ on the $x z$-facet of column $\left(u^{\prime}\right)$, else $\pi_{y z}\left(d^{*}\right)$ and $\pi_{y z}\left(e^{*}\right)$ would cross. Hence $D_{y}^{\prime}>E_{y}^{\prime}$. See Fig. 13. (The case $E_{x}^{\prime}<D_{x}^{\prime}$ would be symmetric, leading to $D_{y}^{\prime}<E_{y}^{\prime}$ and $D^{\prime}$ and $E^{\prime}$ interchanging facets of column $\left(u^{\prime}\right)$.)

Let $\hat{E}$ be the point on $e^{*}$ with the $z$-coordinate $D_{z}^{\prime}$ and let $\hat{D}$ be the point on $d^{*}$ with the $z$-coordinate $E_{z}^{\prime}$. Then $\hat{E}_{x}>D_{x}^{\prime}>\hat{D}_{x}$ and $\hat{D}_{y}>E_{y}^{\prime}>\hat{E}_{y}$.

The remainder of the argument occurs in the $x y$-plane (Fig. 14). We have

$$
\min \left(\hat{D}_{y}-\hat{E}_{y}, \hat{E}_{x}-\hat{D}_{x}\right)<1
$$

since $d\left(\pi_{x y}(\hat{D}), \pi_{x y}\left(e^{*}\right)\right)<1$ and $\pi_{x y}\left(e^{*}\right)$ has positive slope in the $x y$-plane. Hence we have either $d\left(\hat{D}\right.$, column $\left.\left(u^{\prime}\right)\right)<1$ or $d\left(\hat{E}\right.$, column $\left.\left(u^{\prime}\right)\right)<1$. It correspondingly follows that either $d\left(\rho\left(E^{\prime}\right), d^{*}\right) \leq \frac{3}{2}=\kappa$ or $d\left(\rho\left(D^{\prime}\right), e^{*}\right) \leq \frac{3}{2}=\kappa$. 


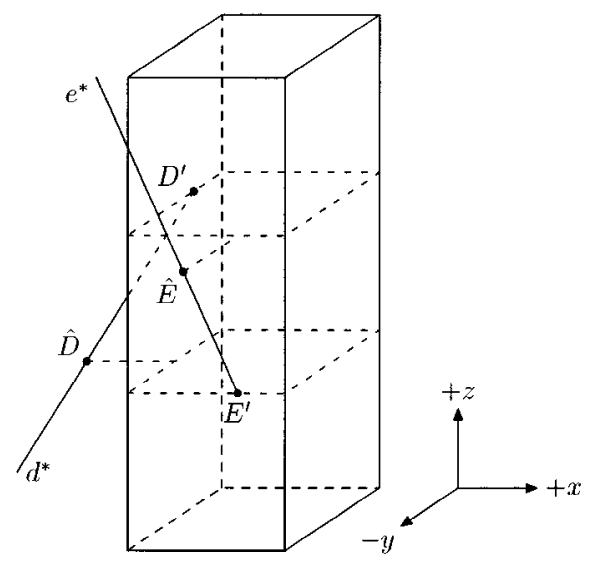

Fig. 13. Case 2 of the proof of Lemma 4.2. Solid outlines are column $\left(u^{\prime}\right)$.

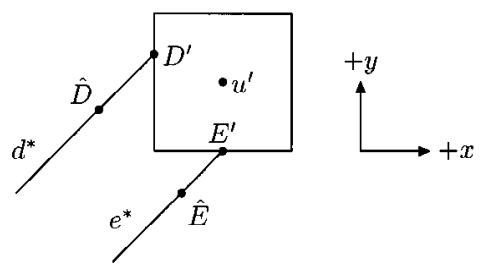

Fig. 14. Case 2 of the proof of Lemma 4.2. Projection onto the $x y$-plane. The solid square is pixel $(u)$. 


\section{Appendix B. Symbol Cross-Reference}

\begin{tabular}{ccl}
\hline Symbol & Section & \\
\hline$d(\cdot, \cdot)$ & 2 & $L_{\infty}$ distance (asymmetric for sets) \\
$d_{\mathrm{H}}(\cdot, \cdot)$ & 2 & Hausdorff distance (symmetric for sets) \\
$\prec, \preceq$ & 2 & Vertical order \\
$\pi_{x y}$ & 2 & Projection on the $x y$ plane \\
$A_{p}$ & 2 & The point of $A$ with $x y$-projection $p$ \\
$P$ & 2 & Original subdivision \\
$Q$ & 2 & Rounded subdivision \\
$\kappa$ & 2 & The distance bound $\left(\frac{3}{2}\right)$ \\
$\sigma$ & 2,6 & Embedding of $P$ into $Q$ \\
$\top, \perp$ & 3 & Symbolic sets at top and bottom of vertical order \\
$\rho$ & 4 & (Naive) three-dimensional snap-rounding \\
$P^{*}$ & 4.1 & Refinement of edges and vertices of $P$ \\
$e^{*}$ & 4.1 & (Refined) edge of $P^{*}$ \\
$T_{f}$ & 4.1 & Triangulation of snap-rounding of projection of $f$ \\
$P_{e}^{*}$ & 4.1 & Edges of $P^{*}$ that project and snap-round to $e$ \\
$P_{v}^{*}$ & 4.1 & Vertices of $P^{*}$ that project and snap-round to $v$ \\
$F_{e}$ & 4.1 & Facets $f$ of $P$ that have $e \in T_{f}$ \\
$F_{\Delta}$ & 4.1 & Facets $f$ of $P$ that have $\Delta \in T_{f}$ \\
$\tau$ & 4.1 & Modified snap rounding on edges \\
$c_{f}(e)$ & 5.2 & Default choice for edges $l_{f \Delta}(e)$ \\
$L_{e}$ & 5.3 & All defined edges over $e$ \\
$V C$ & 6.1 & Vertical carrier \\
$l_{f \Delta}(e)$ & 6.2 & Lifting of edge $e$ of $\Delta$ for facet $f$ \\
$l_{f}(\Delta)$ & 6.2 & Lifting of triangle $\Delta$ for facet $f$ \\
$l_{f}(e)$ & 6.3 & Lifting of edge $e$ for facet $f$ \\
$l_{f}(v)$ & 6.3 & Lifting of vertex $v$ for facet $f$ \\
\hline & &
\end{tabular}

\section{References}

1. H. Brönnimann, M. Yvinec, Efficient exact evaluation of signs of determinants, Proc. Thirteenth Ann. Symp. Comput. Geom, pp. 166-173, 1997.

2. H. Brönnimann, I. Emiris, V. Pan, S. Pion, Computing exact geometric predicates using modular arithmetic with single precision, Proc. Thirteenth Ann. Symp. Comput. Geom, pp. 174-182, 1997.

3. K.L. Clarkson, Safe and effective determinant evaluation, Proc. 33rd Symp. Found. Comput. Sci., pp. 387395, 1992.

4. S. Fortune, Robustness issues in geometric algorithms, in Applied Computational Geometry: Towards Geometric Engineering, pp. 9-14, M. Lin, D. Manocha, eds., Lecture Notes in Computer Science 1148, Springer-Verlag, Berlin, 1996.

5. S. Fortune, Polyhedral modelling with multiprecision integer arithmetic, Computer-Aided Design, 20:123133, 1997.

6. S. Fortune, C. Van Wyk, Static analysis yields efficient exact integer arithmetic for computational geometry, ACM Trans. Graphics, 15(3):223-248, July 1996.

7. M. Goodrich, L. Guibas, J. Herschberger, P. Tanenbaum, Snap-rounding line segments efficiently in two and three dimensions, Proc. Thirteenth Ann. Symp. Comput. Geom, pp. 284-293, 1997. 
8. D. Greene, F. Yao, Finite-resolution computational geometry, Proc. 27th IEEE Symp. Found. Comput. Sci., pp. 143-152, 1986.

9. D. Greene, Integer line segment intersection, Unpublished manuscript.

10. L. Guibas, D. Marimount, Rounding arrangements dynamically, Proc. Eleventh Ann. Symp. Comput. Geom, pp. 190-199, 1995.

11. J. Hobby, Practical segment intersection with finite precision output, Comput. Geom. Theory Appl., to appear.

12. C. Hoffmann, The problems of accuracy and robustness in geometric computation, Computer, 22:31-42, 1989.

13. P. Jaillon, Proposition d'une arithmétique rationnelle paresseuse et d'un outil d'aide à la saise d'objets en synthèse, Ph.D. thesis, Ecole Nationale Superieure des Mine de Saint-Etienee, 1993.

14. M. Karasick, D. Lieber, L. Nackman, Efficient Delaunay triangulation using rational arithmetic, ACM Trans. Graphics 10(1):71-91, 1990.

15. V. Milenkovic, Verifiable implementations of geometric algorithms using finite precision arithmetic, Artificial Intelligence, 37:377-401, 1988.

16. V. Milenkovic, Rounding face lattices in $d$ dimensions, Abstract for Second Canadian Computational Geometry Conference, 1990.

17. V. Milenkovic, Private communication, 1996.

18. V. Milenkovic, Shortest path geometric rounding, Algorithmica, to appear.

19. V. Milenkovic, L. Nackman, Finding compact coordinate representations for polygons and polyhedra, IBM J. Res. Develop. 34(5):752-768, 1990. A version also appeared in Proc. Sixth Ann. Symp. Comput. Geom., pp. 244-252, 1990.

20. J. Shewchuk, Adaptive precision floating-point arithmetic and fast robust geometric predicates, Discrete Comput. Geom., 18(3), 303-363, 1997.

Received September 3, 1997, and in revised form March 29, 1999. 